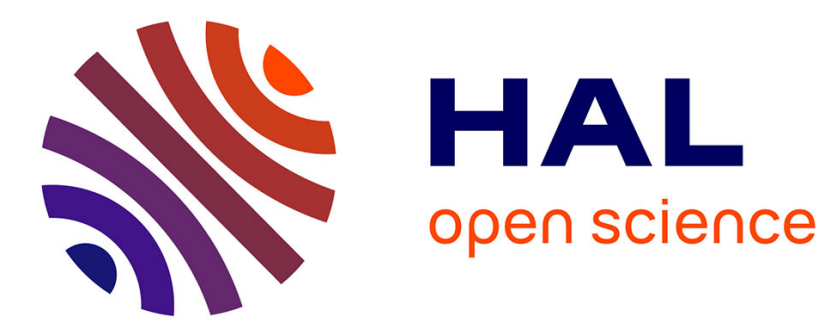

\title{
Vehicle Dynamic Stability Improvements Through Gain-Scheduled Steering and Braking Control
}

Charles Poussot-Vassal, Olivier Sename, Luc Dugard, Sergio Savaresi

\section{To cite this version:}

Charles Poussot-Vassal, Olivier Sename, Luc Dugard, Sergio Savaresi. Vehicle Dynamic Stability Improvements Through Gain-Scheduled Steering and Braking Control. Vehicle System Dynamics, 2011, 49 (10), pp.1597-1621. 10.1080/00423114.2010.527995 . hal-00534721

\section{HAL Id: hal-00534721 \\ https://hal.science/hal-00534721}

Submitted on 10 Nov 2010

HAL is a multi-disciplinary open access archive for the deposit and dissemination of scientific research documents, whether they are published or not. The documents may come from teaching and research institutions in France or abroad, or from public or private research centers.
L'archive ouverte pluridisciplinaire HAL, est destinée au dépôt et à la diffusion de documents scientifiques de niveau recherche, publiés ou non, émanant des établissements d'enseignement et de recherche français ou étrangers, des laboratoires publics ou privés. 


\title{
RESEARCH ARTICLE
}

\section{Vehicle Dynamic Stability Improvements Through Gain-Scheduled Steering and Braking Control}

\author{
C. Poussot-Vassal ${ }^{\text {* }}$, O. Sename ${ }^{\mathrm{b}}$, L. Dugard $^{\mathrm{b}}$ and S.M. Savaresi \\ (Decmber 2009)
}

\begin{abstract}
This paper is concerned with the synthesis of a robust gain-scheduled $\mathcal{H}_{\infty}$ MIMO VDSC (Vehicle Dynamic Stability Controller), involving the steering and braking actuators. This VDSC aims at improving automotive vehicle yaw stability and lateral performances. The contribution of this work is to provide a methodology to synthesize such a controller while taking into account the braking actuator limitations (i.e. the actuator can only brake the rear wheels) and use the steering actuator only if it is necessary (e.g. if the braking system is not efficient enough to ensure vehicle stability, or in case of braking system failure). These objectives are treated in an original way by the synthesis of a parameter dependent controller built in the LPV framework and by the solution of an LMI problem. The proposed solution is coupled with a local ABS strategy to guarantee slip stability and make the solution complete for implementation issues. Nonlinear time and frequency domain simulations on a complex full vehicle model ( which has been validated on a real car), subject to critical driving situations, shows the efficiency and robustness of the proposed solution.
\end{abstract}

Keywords: Vehicle Dynamic Stability; Braking control; Steering control; Control with Saturation; Robust control; LMI; LPV design.

\section{Introduction}

\subsection{Motivations}

Automotive light vehicles are complex systems involving different dynamics. On one side, vertical, roll and pitch behaviors are often related to comfort performances (indeed, roll is also linked to safety characteristics, [1]). On the other hand, safety performances are mainly characterized by the longitudinal, lateral and yaw dynamics. In practice, these two behaviors are often treated in a decoupled may (the first dynamics are often related to suspensions systems, and the second to steering and braking systems). This paper focuses on the safety problem, and more specifically, on lateral and yaw dynamics.

Longitudinal vehicle behaviors have been and still are widely studied trough the rotational wheel and slip dynamic control, leading to the development of ABS (Anti-locking Braking System) strategies [2-5]; mostly, these developments are done using the quarter wheel vehicle model. From the vehicle dynamical point of view, lateral and yaw behaviors are widely considered when handling and safety performances are under interest [6]. On this topic, many papers involve steering

\footnotetext{
Vinci, 32, 20133 Milano, ITALY

* Corresponding author. Email: charles.poussot-vassal@onera.fr

a ONERA - DCSD dpt, 2 Avenue Edouard Belin, 31055 Toulouse, FRANCE

${ }^{\mathrm{b}}$ GIPSA-lab, Control Systems dpt (former LAG), Grenoble University - Grenoble INP, ENSE ${ }^{3}$ - Domaine Universitaire BP46, 38402 Saint Martin d'Hères - Cedex FRANCE

${ }^{\mathrm{c}}$ Dipartimento di Elettronica e Informazione, mOve research team, Politecnico di Milano, Piazza L. da 
control [7-11], or braking control [12, 13], but a few use both actuators [14]. Indeed, collaborative control of different kind of actuators is an issue in global chassis control and safety improvements [15-19].

In [20], authors show that the join use of the braking and steering systems highly enhances lateral performances and ground vehicles safety properties. To achieve this objective using different actuators, nice recent developments mostly involve MPC $[8,12,21]$ or nonlinear $[22]$ techniques which often results to be highly demanding from a computational point of view.

Nevertheless, when both steering and braking actuators are used, the following limitations are rarely considered during the synthesis step:

- When braking system is used for vehicle stability control, the synthesized MIMO controller does not always handle the fact that the braking actuator can only generate positive braking torque (since, negative torque would means that the wheel is accelerated, which is not possible on most of commercial cars). Indeed, this problem is often treated trough differential braking which limits may lead to unrealistic braking force demand.Hence, closed loop performances are usually deteriorated due to this limitation and a braking repartition law is then required.

- Even if the steering system may enhance the vehicle dynamical behavior, it is unlikely for the driver that the steering wheel act too much when riding (driving comfort). As a consequence, steering should act in a given frequency range and only if it is required (see [10]).

\subsection{Contributions and structure of the paper}

In [23], the vehicle lateral stability is enhanced while using a parameter that activates the steering action when the braking systems is not efficient enough (but without taking into account, in the synthesis, the braking limitations). In [24], the braking actuator limitations are handled trough the use of a scheduling parameter but steering actuator is always used.

The contribution of this paper is to propose a design methodology to synthesize a VDSC that enhances yaw stability and lateral dynamics trough the use of rear braking and active front steering control, while handling limitations and requirements described above.

Compared to the previous authors results (see [23] and [24]), the main contributions are to provide an unifying LPV controller structure, consistent performances weights (inspired from [10] results), together with an LMI solution to design a controller ensuring $\mathcal{H}_{\infty}$ performances while handling the braking actuator constraints in an original way and activating the steering actuator only when it is required.

More than a simple controller, the interest of the proposed VDSC is that it provides a hierarchy to the local controllers and actuators. Therefore, the aim of the VDSC is to be used as a higher level controller to provide references to local controllers (as illustrated on Figure 1).

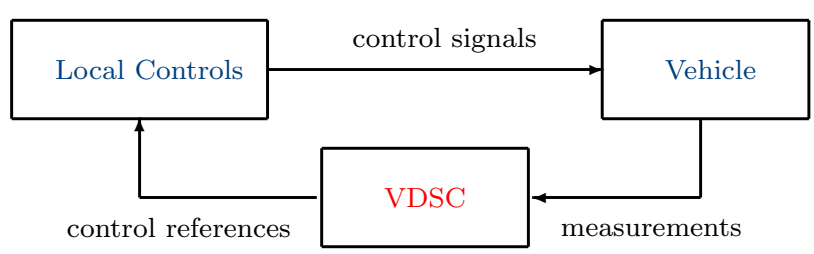

Figure 1. Simplified control scheme.

When dangerous situations are detected, the VDSC gives a consistent torque 


\begin{tabular}{llcl}
\hline Symbol & Value & Unit & Meaning \\
\hline \hline$m$ & 1535 & $\mathrm{~kg}$ & vehicle mass \\
$m_{r}$ & 648.3 & $\mathrm{~kg}$ & vehicle rear mass \\
$I_{z}$ & 2149 & $\mathrm{~kg} \cdot \mathrm{m}^{2}$ & vehicle yaw inertia \\
$C_{f}$ & 40000 & $\mathrm{~N} / \mathrm{rd}$ & linear lateral tire front cornering stiffness \\
$C_{r}$ & 40000 & $\mathrm{~N} / \mathrm{rd}$ & linear lateral tire rear cornering stiffness \\
$l_{f}$ & 1.4 & $\mathrm{~m}$ & distance COG - front axle \\
$l_{r}$ & 1 & $\mathrm{~m}$ & distance COG - rear axle \\
$t_{r}$ & 1.4 & $\mathrm{~m}$ & rear axle length \\
$R$ & 0.3 & $\mathrm{~m}$ & tire radius \\
$T_{b_{\max }}$ & 1200 & $\mathrm{Nm}$ & maximal braking value \\
$g$ & 9.81 & $\mathrm{~m} / \mathrm{s}^{2}$ & gravitational constant \\
\hline$\mu$ & {$[2 / 5 ; 1]$} & - & tire/road contact friction interval \\
$v$ & {$[50 ; 120]$} & $\mathrm{km} / \mathrm{h}$ & vehicle velocity interval \\
\hline
\end{tabular}

Table 1. Linear bicycle Renault Mégane Coup parameters.

reference (e.g. positive) to the braking system (that avoids slipping thanks to the local ABS controller), and, if the braking system is not efficient enough to stabilize the vehicle (e.g. in case of low adherence, braking failure, etc.), the steering system is activated to handle the dynamical problem.

As long as the proposed solution does not require either an on-line optimization procedure nor a matrix inversion, and uses available measured signals and actuators, the structure also shows to be easy to implement on commercial cars. The proposed solution exploits one of the system structural property to modify the controller output behavior (using an LPV design), making it more robust and efficient in different situations. This is done in the framework of MIMO systems and proof of stability and performances are preserved for the entire set of varying parameters.

The paper is structured as follows: Section 1 provides introductive elements, notations and motivations. Section 2 introduces the full nonlinear vehicle model (which has been validated on a real car [25]), used for the validation, performance analysis and evaluation. Section 3 presents the model used for synthesis together with the considered actuator models. The main contribution of the paper, namely, the LMI based synthesis of the MIMO gain-scheduled VDSC using steering and braking systems (handling braking and steering actuator limitations, and adjusted according to the driving situation) is presented in Sections 4 (problem formulation) and 5 (controller synthesis). In Section 6, performance are analyzed and compared to other design techniques trough frequency and time simulations performed on the complex nonlinear full vehicle model presented in Section 2. Conclusions and discussions are given in Section 7.

\subsection{Paper notations}

Throughout the paper, the following standard notations will be adopted: $A+\star^{T}$ stands for $A+A^{T}$, the LPV induced $\mathcal{L}_{\infty}$ to $\mathcal{L}_{\infty}$ norm will be denoted as the $\mathcal{H}_{\infty}$ norm. The index $i=\{f, r\}$ and $j=\{l, r\}$ are used to identify vehicle front, rear and left, right vehicle corner positions respectively. The Laplace variable is denoted as 's'. Table 1 summarizes the notations and values used in the paper to define the linear bicycle model. Parameters of the complete full nonlinear model are given in [25]. 


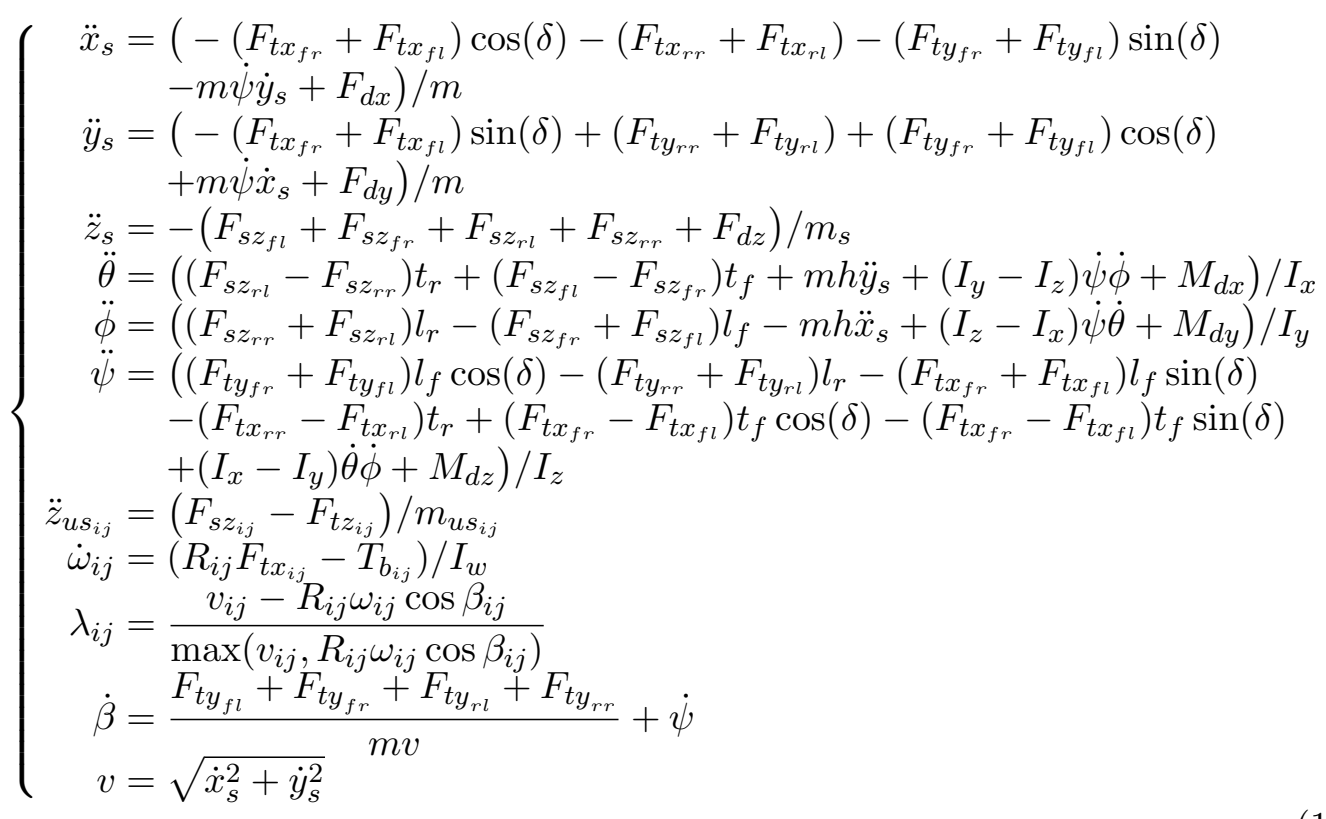

\section{Simulation model: Full vehicle}

In this paper, the full vehicle model presented in [25] is used for simulation and validation purpose. This model and its parameters has been validated on a real Renault Mégane vehicle ${ }^{1}$. For sake of completeness, such a model is recalled thereafter but interested reader should refer to [25] for more details on parameters values and for model validation on real experiments.

Note that the main interest in using the full nonlinear vehicle model is that is allows to take into consideration nonlinear load transfer, nonlinear fast dynamics entering in the tire force description, and consequently, in the global chassis dynamics, especially in dangerous driving situations in which we are interested in.

The full vehicle model is defined by the nonlinear dynamical equations referred in (1).

It reproduces the longitudinal $\left(x_{s}\right)$, lateral $\left(y_{s}\right)$, vertical $\left(z_{s}\right)$, roll $(\theta)$, pitch $(\phi)$ and yaw $(\psi)$ dynamics of the chassis. It also models the vertical and rotational motions of the wheels $\left(z_{u s_{i j}}\right.$ and $\omega_{i j}$ respectively), the slip ratios $\left(\lambda_{i j}\right)$ and the center of gravity side slip angle $(\beta)$ dynamics as a function of the tires and suspensions forces. The forces, describing the entire vehicle dynamics are given thereafter.

\subsection{Suspensions model $\left(\boldsymbol{F}_{s z_{i j}}\right)$}

Suspensions are usually modeled by a spring and a damping element. In real vehicles, their characteristics are highly nonlinear and may present hysteresis phenomena. Here, as long as we mainly focus on the longitudinal, lateral and yaw behaviors, without loss of generality, static non linear stiffness and damping coefficients will be considered (see e.g. [26, 27]). Therefore, the suspension model adopted here is

\footnotetext{
${ }^{1}$ Thanks to M. Basset, G. Pouly and C. Lamy, from the MIAM research team for their kind help in the
} validation phase. 
simply given by:

$$
F_{s z_{i j}}=F_{k_{i j}}\left(z_{s_{i j}}-z_{u s_{i j}}\right)+F_{c_{i j}}\left(\dot{z}_{s_{i j}}-\dot{z}_{u s_{i j}}\right)
$$

where $F_{k_{i j}}$ and $F_{c_{i j}}$ are nonlinear static functions of $\left(z_{s_{i j}}-z_{u s_{i j}}\right)$ and $\left(\dot{z}_{s_{i j}}-\dot{z}_{u s_{i j}}\right)$ respectively.

\subsection{Longitudinal tire model $\left(\boldsymbol{F}_{t x_{i j}}\right)$}

The longitudinal Burkhardt model is given by (3) (see also $[2,28]$ ),

$$
F_{t x_{i j}}=\left(\mu_{1}\left(1-e^{-\lambda_{i j} \mu_{2}}\right)-\lambda_{i j} \mu_{3}\right) F_{n_{i j}}
$$

where $\left[\mu_{1}, \mu_{2}, \mu_{3}\right]$ describes the longitudinal road friction shape according to the considered road condition and $F_{n_{i j}}=g\left(m_{u s_{i j}}+m_{s} / 4\right)-\left(F_{t z_{i j}}+F_{s z_{i j}}\right)$ holds for the normal load at each corner of the vehicle.

\subsection{Lateral tire model $\left(\boldsymbol{F}_{t y_{i j}}\right)$}

The lateral tire forces are given by formulae (4)

$$
F_{t y_{i j}}=D \sin \left(C \arctan \left(B(1-E) \beta_{i j}+E \arctan \left(B \beta_{i j}\right)\right)\right) e^{-6\left|\lambda_{i j}\right|^{5}}
$$

where,

$$
\left\{\begin{array}{l}
\beta_{f j}=-\beta-l_{f} \frac{\dot{\psi}}{v}+\delta \\
\beta_{r j}=-\beta+l_{r} \frac{\dot{\psi}}{v}
\end{array}\right.
$$

are the front and rear side slip angle respectively. Then $B=(2-\mu) b_{t}, C=$ $(5 / 4-\mu / 4) c_{t}, D=d_{t} \mu$ and $E=e_{t}$ are the lateral tire parameters, function of $\mu \in[0 ; 1]$, the tire/road adhesion coefficient. Additionally, $e^{-6\left|\lambda_{i j}\right|^{5}}$ is used to model the fact that lateral friction forces are decreasing when slipping occurs (e.g. when vehicle is slipping, it is no longer manoeuvrable) then, $\lim _{\lambda_{i j} \rightarrow|1|} F_{t y_{i j}}=0$.

\subsection{Vertical tire model $\left(\boldsymbol{F}_{t z_{i j}}\right)$}

Finally, the vertical forces are linearly described by (6),

$$
F_{t z_{i j}}=k_{t_{i j}}\left(z_{u s_{i j}}-z_{r_{i j}}\right)+c_{t_{i j}}\left(\dot{z}_{u s_{i j}}-\dot{z}_{r_{i j}}\right)
$$

where $k_{t}$ and $c_{t}$ are the tire stiffness and damping coefficients respectively.

\section{Synthesis model: Bicycle \& Actuators}

The previous model (given in Section 2) is used in Section 6 to evaluate the controller performances. In this Section, the model used for synthesis is presented together with the considered actuators. This model is a modified version of the bicycle model (as the one described in $[7,11]$ ). 


\subsection{Extended bicycle model}

Since this paper aims at improving vehicle safety properties in critical situations to avoid loss of manoeuvrability, the main nonlinear dynamics under interest are simplified as:

$$
\left\{\begin{aligned}
m v \dot{\beta} & =F_{t y_{f}}+F_{t y_{r}}+m v \dot{\psi} \\
I_{z} \ddot{\psi} & =l_{f}\left(-F_{t x_{f}} \sin (\delta)+F_{t y_{f}} \cos (\delta)\right)-l_{r} F_{t y_{r}}-\Delta F_{t x_{r}} t_{r}+M_{d z}
\end{aligned}\right.
$$

where $F_{t y_{f}}=F_{t y_{f l}}+F_{t y_{f r}}, F_{t y_{r}}=F_{t y_{r l}}+F_{t y_{r r}}$ and $\Delta F_{t x_{r}}=F_{t x_{r l}}-F_{t x_{r r}}$ are the lateral front, rear tire lateral forces and longitudinal rear differential forces respectively. In the nonlinear model, these forces are highly nonlinear and depend, among other, on the sideslip angle $(\beta)$ and the slip ratio $(\lambda)$ dynamics. $v=\sqrt{\dot{x}_{s}^{2}+\dot{y}_{s}^{2}}$ denotes the longitudinal vehicle speed. $\Delta F_{t x_{r}}$ is the differential rear braking force, which depends on the the braking torques $T_{b_{r j}} . \delta$ and $M_{d z}$ denote the steering angle and the yaw moment disturbance respectively. More specifically, $\delta=\delta^{d}+\delta^{+}$, where $\delta^{d}$ is the angle provided by the driver and $\delta^{+}$, the additive steering angle provided by the controller (see Section 5). Assuming that low slip value are preserved (i.e. $\lambda_{i j}$ low), $\Delta F_{t x_{r}}$ may be rewritten as,

$$
\begin{aligned}
\Delta F_{t x_{r}} & =F_{t x_{r l}}-F_{t x_{r r}} \\
& =\frac{\mu R m_{r} g}{2}\left(T_{b_{r l}}-T_{b_{r r}}\right)
\end{aligned}
$$

consequently, (7) may be rewritten as follows,

$$
\left\{\begin{aligned}
m v \dot{\beta} & =F_{t y_{f}}+F_{t y_{r}}+m v \dot{\psi} \\
I_{z} \ddot{\psi} & =l_{f}\left(-F_{t x_{f}} \sin (\delta)+F_{t y_{f}} \cos (\delta)\right)-l_{r} F_{t y_{r}}-\mu R m_{r} g\left(T_{b_{r l}}-T_{b_{r r}}\right) t_{r}+M_{d z}
\end{aligned}\right.
$$

Since the proposed design is done in the linear control framework, the bicycle model (10), denoted as $\Sigma$, obtained from linearization of (9) at a given nominal velocity, is used for synthesis purpose.

$$
\begin{aligned}
{\left[\begin{array}{c}
\dot{\beta} \\
\ddot{\psi}
\end{array}\right]=} & {\left[\begin{array}{cc}
\mu \frac{-C_{f}-C_{r}}{m v} & 1+\mu \frac{l_{r} C_{r}-l_{f} C_{f}}{m v^{2}} \\
\mu \frac{l_{r} C_{r}-l_{f} C_{f}}{I_{z}} & \mu \frac{-l_{f}^{2} C_{f}-l_{r}^{2} C_{r}}{I_{z} v}
\end{array}\right]\left[\begin{array}{c}
\beta \\
\dot{\psi}
\end{array}\right] } \\
+ & {\left[\begin{array}{cccc}
\frac{C_{f}}{m v} & 0 & 0 & 0 \\
\frac{l_{f} C_{f}}{I_{z}} & \frac{1}{I_{z}}-\frac{\mu m_{r} g R t_{r}}{2 I_{z}} & \frac{\mu m_{r} g R t_{r}}{2 I_{z}}
\end{array}\right]\left[\begin{array}{c}
\delta \\
M_{d z} \\
T_{b_{r l}} \\
T_{b_{r r}}
\end{array}\right] }
\end{aligned}
$$

The linear bicycle model is obtained through the following assumptions:

- Low sideslip angles: $|\beta|<7 \mathrm{deg}$

- Low longitudinal slip ratios: $|\lambda|<0.1$

- Low steering angles: $\cos (\delta) \simeq 1 \mathrm{rd}$

Note that this model is similar to the well known model presented in [7], but here, it is extended to include the rear braking torque inputs. For synthesis purpose, $v=30 \mathrm{~m} / \mathrm{s}$ and $\mu=1$. In the validation section, other conditions will be considered to show that performances are kept. 


\subsection{Actuator models}

In the paper, the considered actuators are two rear EMB (Electro Mechanical Braking) systems, which allow to provide continuously variable modulation rear braking torques, and a front AS (Active Steering) system, that is simply an electric motor mounted on the column direction. These actuators will be modeled as first order low-pass transfer functions:

- As braking system, we consider EMB actuators, providing a continuously variable braking torque. The model is given by:

$$
\dot{T}_{b_{r j}}=2 \pi \varpi\left(\widetilde{T_{b_{r j}}^{*}}-T_{b_{r j}}\right)
$$

where, $\varpi=10 \mathrm{~Hz}$ is the actuator cut-off frequency, $\widetilde{T_{b_{r j}}^{*}}$ and $T_{b_{i j}}$ are the local braking controller and actuator outputs respectively. Note that in this paper, only the rear braking system is used to avoid coupling phenomena occurring with the steering system. Moreover, rear brakes more affect the vehicle yaw behavior than the front one. The braking torque is bounded as $T_{b_{r j}} \in \mathcal{T}_{b}$, where $\mathcal{T}_{b}:=\left\{T_{b} \in \mathbb{R}: 0 \leq T_{b} \leq T_{b_{\max }}\right\}$

- As active steering system (AS), an active actuator providing an additional steering angle is considered. Such actuator is modeled as:

$$
\dot{\delta}^{+}=2 \pi \kappa\left(\delta^{*}-\delta^{+}\right)
$$

where, $\kappa=10 \mathrm{~Hz}$ is the actuator cut-off frequency, $\delta^{*}$ and $\delta^{+}$are the steering controller and actuator outputs respectively.

\subsection{Limitations of the linear model}

Since the linear model is valid under specific assumptions, after synthesis, all validations will be done on the full vehicle nonlinear model which reproduces all the most critical vehicle dynamics (for more details, see [25]):

- Nonlinear slip and sideslip dynamics

- Nonlinear load transfer

- Vertical, roll and pitch dynamics

- Longitudinal, lateral and yaw coupled dynamics

In the validation step presented in Section 6, robustness of the controller is illustrated by using frequency results obtained on the nonlinear full vehicle model.

\section{Main result: MIMO Gain-Scheduled VDSC (problem formulation)}

In this Section, the problem formulation of the main result of this paper is presented. First, the performance objectives and the global control scheme (architecture of the control structure) are given, then, the generalized control scheme together with the controller particular structure are described.

\subsection{Performance objective}

The objectives to be achieved are the following:

(1) To reduce the yaw rate error w.r.t. a reference provided by a reference model 
(which is a bicycle model where parameters may be adjusted in order to obtain comfort or sport driving performances), without using the sideslip measurement (which is practically badly known).

(2) To reduce roll velocity (indirect effect).

(3) To ensure that the braking control signal is always achievable by the considered actuators.

(4) To make the system efficient in critical situation (low road adherence) or in actuators failure case.

\subsection{General control scheme}

To achieve these requirements, the following general control structure scheme of the braking and steering controller is proposed (see Figure 2). Note that this scheme will be used later for nonlinear validations in Section 6 .

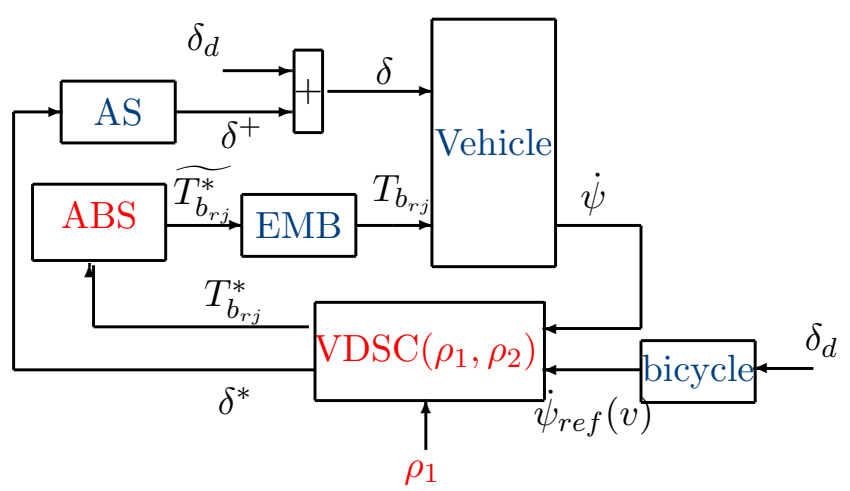

Figure 2. Global control scheme.

In Figure 2 the proposed global control structure includes the following blocks which will be described in the following subsections:

- Vehicle \& Actuators (AS \& EMB): are the full nonlinear vehicle and actuator models (see Section 2).

- VDSC: is the proposed brake and steering controller providing the desired braking torque $\left(T_{b_{r j}}^{*}\right)$ and the additive steering angle $\left(\delta^{*}\right)$. It is feeded by $e_{\dot{\psi}}=\dot{\psi}_{r e f}(v)-\dot{\psi}$ and scheduled by $\rho_{2}$, a function of $e_{\dot{\psi}}$ and $\rho_{1}$, the monitoring parameter (the scheduling variables are described in more details in subsection 4.4). $\dot{\psi}_{r e f}(v)$ is obtained by a simple bicycle model similar to the one presented in Section 3, equation (9), function of the vehicle velocity, and parameterized as a neutral vehicle. Note that it could be interesting to vary some of the parameters to tune the reference vehicle toward a sport/neutral/comfort behavior (but since it is not the topic of this paper, this point is not covered in this paper).

- ABS: is the local ABS control, implemented on each of the rear wheels, that is activated to avoid slipping, providing $\widetilde{T_{b_{r j}}^{*}}$, the braking torque, according to the set point $T_{b_{r j}}^{*}$ given by the VDSC controller (this design is based on recently published results, see [5] and subsection 5.4). 


\subsection{Gain-scheduled Vehicle Dynamic Stability Controller: Generalized plant \& Weighting functions}

To synthesize the so called gain-scheduled VDSC, the $\mathcal{H}_{\infty}$ control approach is used. In the following the generalized synthesis plant, the performance weighting functions, the LPV controller structure are presented [29]. The generalized plant is given on Figure 3.

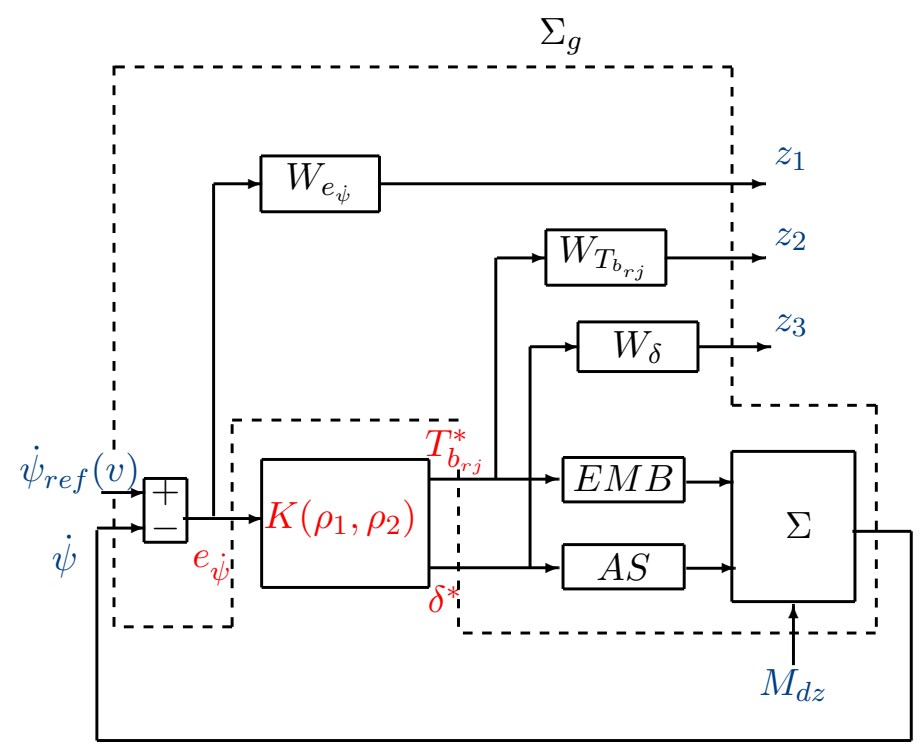

Figure 3. Generalized plant model.

According to the standard problem defined in Figure 3 the following systems and signals are defined, to characterize the performance objectives and actuator frequency limitations:

- $\Sigma$, EMB and AS are the extended bicycle and actuators LTI models respectively (see Section 3).

- $K\left(\rho_{1}, \rho_{2}\right)$ is the controller to be synthesized (see next subsection).

- $z_{1}$, yaw rate error output signal, which represents the tracking error performance, is defined by:

$$
W_{e_{\psi}}=\frac{1}{2 G_{e}} \frac{s G_{e} / 2 \pi f_{1}+1}{s / 2 \pi f_{1}+1}
$$

where $f_{1}=1 \mathrm{~Hz}$ is the cut-off frequency of the high pass filter. $G_{e}=0.1$ is the attenuation level for low frequencies $\left(f<f_{1}\right)$. In this case 0.1 means that the static tracking error should be lower than $10 \%$.

- $z_{2}$, the braking control signal attenuation, is defined by:

$$
W_{T_{b_{r j}}}=10^{-4} \frac{s / 2 \pi f_{2}+1}{s / \alpha 2 \pi f_{2}+1}
$$

where $f_{2}=10 \mathrm{~Hz}$ is the braking actuator bandwidth and $\alpha=100$. These parameters are chosen to handle braking actuator limitations (dynamic limits). 
- $z_{3}$, the steering control signal attenuation, is defined by:

$$
\begin{aligned}
W_{\delta} & =G_{\delta}^{0} \frac{\left(s / 2 \pi f_{3}+1\right)\left(s / 2 \pi f_{4}+1\right)}{\left(s / \alpha 2 \pi f_{4}+1\right)^{2}} \\
G_{\delta}^{0} & =5.10^{-3} \frac{\left(\Delta_{f} / \alpha 2 \pi f_{4}+1\right)^{2}}{\left(\Delta_{f} / 2 \pi f_{3}+1\right)\left(\Delta_{f} / 2 \pi f_{4}+1\right)} \\
\Delta_{f} & =2 \pi\left(f_{4}+f_{3}\right) / 2
\end{aligned}
$$

where $f_{4}=10 \mathrm{~Hz}$ is the steering actuator bandwidth and $f_{3}=1 \mathrm{~Hz}$ is lower limit of the actuator intervention. This filter is designed in order to allow the steering system to act only in $\left[f_{3}, f_{4}\right]$ frequency range. Outside of this frequency range, the filter rolls off. Between these frequencies, and more specifically, at $\Delta_{f} / 2$, the steering action is allowed. The interest of such complex filter is to allow the steering system to act at frequencies the driver is not able to provide, while handling the actuator limitations. This filter design is inspired from $[10,30]$ where a filter $(Q)$ of the same shape was used to limit the steering bandwidth action. But in $[10,30]$ the filter is added a posteriori and synthesis only involves steering actuator. Here the filter is included in the design specifications.

These weighting functions are recalled in the sensitivity function plot given in Figures 4, 5 and 6 . The generalized control scheme obtained (from Figure 3) is given by:

$$
\Sigma_{g}:\left\{\begin{array}{l}
\dot{\xi}(t)=A \xi(t)+B_{1} w(t)+B_{2} u(t) \\
z(t)=C_{1} \xi(t)+D_{11} w(t)+D_{12} u(t) \\
y(t)=C_{2} \xi(t)+D_{21} w(t)
\end{array}\right.
$$

where,

$$
\begin{aligned}
w(t) & =\left[\dot{\psi}_{r e f}(v)(t), M_{d z}(t)\right] \quad \text { the exogenous input signals } \\
u(t) & =\left[\delta^{*}(t), T_{b_{r l}}^{*}(t), T_{b_{r r}}^{*}(t)\right] \text { the control input signals } \\
y(t) & =e_{\dot{\psi}}(t) \quad \text { the signal measurement } \\
z(t) & =\left[z_{1}(t), z_{2}(t), z_{3}(t)\right] \quad \text { the controlled outputs signals }
\end{aligned}
$$

where $\xi(t)$ is the concatenation of the linearized vehicle model, actuators and weighting function state variables, which takes its values in $\Xi \in \mathbb{R}^{n}, z(t)$ the performance output which takes its values in $Z \in \mathbb{R}^{n_{z}}, w(t)$ the weighted input which takes its values in $W \in \mathbb{R}^{n_{w}}, y(t)$ the measured signal which takes its values in $Y \in \mathbb{R}^{n_{y}}, u(t)$ the control signal which takes its values in $U \in \mathbb{R}^{n_{u}}$. Then, $A \in \mathbb{R}^{n \times n}, B_{1} \in \mathbb{R}^{n \times n_{w}}, B_{2} \in \mathbb{R}^{n \times n_{u}}, C_{1} \in \mathbb{R}^{n_{z} \times n}, D_{11} \in \mathbb{R}^{n_{z} \times n_{w}}, D_{12} \in \mathbb{R}^{n_{z} \times n_{u}}$, $C_{2} \in \mathbb{R}^{n_{y} \times n}$ and $D_{21} \in \mathbb{R}^{n_{y} \times n_{w}}$ are known matrices. In this case, $n=8, n_{w}=2$, $n_{u}=3, n_{z}=3$ and $n_{y}=1$.

\subsection{Gain-scheduled Vehicle Dynamic Stability Controller: LPV controller structure and parameters description}

Usually, when such kind of stability control is to be synthesized, the controller is built so that we obtain the linear closed-loop performances, without taking into account the fact that the braking torque must be positive (as in [23]). It results in a controller which may provide a negative torque (equivalent to an acceleration), which is, practically impossible. Therefore, a saturation on the braking signal is often applied and performances are a posteriori validated. Unfortunately, as illustrated in Section 6, this kind of design may lead to high forces and to undesirable 
vehicle behavior. Moreover, the steering action should be shut off/on if necessary. To handle theses constraints, a particular LPV controller structure is proposed here. Let first introduce the considered varying parameters:

- $\rho_{1} \in[0 ; 1]$ : used to activate the steering action (if necessary).

- $\rho_{2} \in\{0 ; 1\}$ : used to ensure positive braking torque.

Note that both parameters are design parameters since they are not related to one of the vehicle model nonlinearity. These parameters will be detailed more precisely in the following sections.

Consequently, the controller structure is fixed, but a parameter dependency on the control output matrix is introduced. Accordingly, the following controller structure is chosen (denoted as $\left.K\left(\rho_{1}, \rho_{2}\right)\right)$ :

$$
\left\{\begin{aligned}
\dot{x}_{c}(t) & =A_{c}\left(\rho_{1}, \rho_{2}\right) x_{c}(t)+B_{c}\left(\rho_{1}, \rho_{2}\right) e_{\dot{\psi}}(t) \\
{\left[\begin{array}{c}
\delta^{*}(t) \\
T_{b_{r l}}^{*}(t) \\
T_{b_{r r}}^{*}(t)
\end{array}\right] } & =\underbrace{\left[\begin{array}{ccc}
\rho_{1} & 0 & 0 \\
0 & \rho_{2} & 0 \\
0 & 0 & 1-\rho_{2}
\end{array}\right] C_{c}^{0}\left(\rho_{1}, \rho_{2}\right)}_{C_{c}\left(\rho_{1}, \rho_{2}\right)} x_{c}(t)
\end{aligned}\right.
$$

where $x_{c}(t)$, the controller state, takes its values in $\Xi_{c} \in \mathbb{R}^{n}, u(t)=$ $\left[\delta^{*}(t) T_{b_{r l}}^{*}(t) T_{b_{r r}}^{*}(t)\right]^{T}$ and $y(t)=e_{\dot{\psi}}(t)$.

Considering this specific controller structure, and accordingly to the parameter variation range, the following controller properties holds:

- $\rho_{1} \in[0,1]$ allows to continuously (de)activates the steering action. The aim of this parameter is to schedule the VDSC to activate the steering system when it is required. More specifically:

- when $\rho_{1}=0$, the $\delta^{*}(t)$ signal is set to zero (no steering action).

- when $\rho_{1}=1$, the $\delta^{*}(t)$ signal is activated (steering action is allowed).

- in between values provide intermediate steering activation. As a consequence, the steering system may be smoothly activated through this parameter. The activating rule is not covered in this paper, but many mechanisms may be used: e.g. a braking system fault detection (see e.g. [23]), a critical situation monitor (see e.g. [1]) or vehicle dynamics monitoring (see e.g. [31-33]).

Therefore, the $\rho_{1}$ parameter is considered as a performance parameter that can be adjusted on-line by a mechanism. Note that $\rho_{1}$ may continuously vary between 0 and 1.

- $\rho_{2} \in\{0 ; 1\}$ selects the activated braking actuator. According to its value (either 0 or 1$)$ the controller output will vary. More specifically:

- when $\rho_{2}=1$, the $T_{b_{r r}}^{*}(t)$ signal is set to zero

- when $\rho_{2}=0$, the $T_{b_{r l}}^{*}(t)$ signal is set to zero

Then, to cope with the yaw rate tracking performance and braking selection, $\rho_{2}$ is chosen as:

$$
\rho_{2}(t)=\operatorname{sat}_{[0,1]}\left[\operatorname{sign}\left(e_{\dot{\psi}}(t)\right)\right]
$$

Hence,

$$
\begin{aligned}
& e_{\dot{\psi}}(t)>0 \Rightarrow \rho_{2}=1 \text { (rear left brake is activated) } \\
& e_{\dot{\psi}}(t) \leq 0 \Rightarrow \rho_{2}=0 \text { (rear right brake is activated) }
\end{aligned}
$$

As a matter of fact, by scheduling $\rho_{2}$ with the rule (19), it is ensured that the 
braking signal sent by the controller is always positive, which is consistent with reality. Therefore, the controller exploits a property of the system to handle this constraint. Note that $\rho_{2}$ only takes two values, either 0 or 1 .

On table 2, the activated controller output according to the parameters values is summarized.

\begin{tabular}{c|c||c|c}
\hline$\rho_{1}$ & $\rho_{2}$ & Actuators activated & Actuators deactivated \\
\hline \hline 0 & 0 & $T_{b_{r r}}^{*}(t)$ & $T_{b_{r l}}^{*}(t)$ and $\delta^{*}(t)$ \\
& 1 & $T_{b_{r l}}^{*}(t)$ & $T_{b_{r r}}^{*}(t)$ and $\delta^{*}(t)$ \\
\hline 1 & 0 & $\delta^{*}(t)$ and $T_{b_{r r}}^{*}(t)$ & $T_{b_{r l}}^{*}(t)$ \\
& 1 & $\delta^{*}(t)$ and $T_{b_{r l}}^{*}(t)$ & $T_{b_{r r}}^{*}(t)$ \\
\hline
\end{tabular}

Table 2. $\left\{\rho_{1}, \rho_{2}\right\}$-parameter configurations and actuators activation.

The interest of this LPV structure is that during the synthesis step, the controller 'knows' that only one single brake is available at each time and that the steering actuator is not always available. As a consequence, controller will be designed so that internal stability and closed-loop performances are guaranteed for the all set of varying parameters. In Section 5 , the LMI solution of the $\mathcal{H}_{\infty}$ problem is given for this specific problem.

\section{Main result: MIMO Gain-Scheduled VDSC (controller synthesis)}

In this Section, the LMI based synthesis gain-scheduled VDSC and its implementation form are presented. The local ABS strategy is also briefly recalled (based on $[5])$.

\subsection{LMI solution of the LPV problem}

The generalized system $\Sigma_{g}$ (system and actuators models) is LTI, but the controller structure is LPV. To find a stabilizing controller, ensuring $\mathcal{H}_{\infty}$ performances, the following propositions has to be satisfied. For any system, the $\mathcal{H}_{\infty}$ control synthesis is a disturbance attenuation problem. It consists in finding a stabilizing controller that minimizes the impact of the input disturbances $w(t)$ on the controlled output $z(t)$. In the case of the LPV $\mathcal{H}_{\infty}$ control, this impact is measured thanks to the induced $\mathcal{L}_{2}-\mathcal{L}_{2}$ norm. This problem is represented on Figure 3.

The $\mathcal{H}_{\infty}$ control synthesis solution for LPV systems is extended from the LTI one. The following two propositions solve the $\mathcal{H}_{\infty}$ "LPV VDSC" problem using a polytopic approach (the first one is related to the feasibility, and the second one, to the controller reconstruction). For more details on $\mathcal{H}_{\infty}$ and LPV modeling and control, interested reader should refer to very interesting references [34-40].

\subsubsection{Proposition: Feasibility $-\mathcal{H}_{\infty}$ LMI based VDSC design.}

Let consider the system interconnection on Figure 3, where $\Sigma_{g}$ is defined by the state space representation given in (16) and $\left\{\rho_{1}, \rho_{2}\right\} \in\left[\underline{\rho_{1}}, \overline{\rho_{1}}\right] \times\left[\underline{\rho_{2}}, \overline{\rho_{2}}\right]$. There exist a full order gain-scheduled dynamical output feedback controller $\left(K\left(\rho_{1}, \rho_{2}\right)\right)$ of the form (18), that minimizes the LPV polytopic $\mathcal{L}_{2}-\mathcal{L}_{2}$ induced norm if there exist 


$$
\left[\begin{array}{ccc}
A \mathbf{X}+B_{2} \widetilde{\mathbf{C}}\left(\rho_{1}, \rho_{2}\right)+(\star)^{T} & (\star)^{T} & (\star)^{T}(\star)^{T} \\
\widetilde{\mathbf{A}}\left(\rho_{1}, \rho_{2}\right)+A^{T} & \mathbf{Y} A+\widetilde{\mathbf{B}}\left(\rho_{1}, \rho_{2}\right) C_{2}+(\star)^{T} & (\star)^{T}(\star)^{T} \\
B_{1}^{T} & B_{1}^{T} \mathbf{Y}+D_{21}^{T} \widetilde{\mathbf{B}}\left(\rho_{1}, \rho_{2}\right)^{T} & -\gamma I(\star)^{T} \\
C_{1} \mathbf{X}+D_{12} \widetilde{\mathbf{C}}\left(\rho_{1}, \rho_{2}\right) & C_{1} & D_{11}-\gamma I
\end{array}\right] \prec 0 \text { and }\left[\begin{array}{cc}
\mathbf{X} & I \\
I & \mathbf{Y}
\end{array}\right] \succ 0
$$

$$
\left\{\begin{array}{l}
C_{c}\left(\rho_{1}, \rho_{2}\right)=\widetilde{C}\left(\rho_{1}, \rho_{2}\right) M^{-T} \\
B_{c}\left(\rho_{1}, \rho_{2}\right)=N^{-1} \widetilde{B}\left(\rho_{1}, \rho_{2}\right) \\
A_{c}\left(\rho_{1}, \rho_{2}\right)=N^{-1}\left(\widetilde{A}\left(\rho_{1}, \rho_{2}\right)-Y A X-N B_{c}\left(\rho_{1}, \rho_{2}\right) C_{2} X-Y B_{2} C_{c}\left(\rho_{1}, \rho_{2}\right) M^{T}\right) M^{-T}
\end{array}\right.
$$

symmetric matrices $X, Y \in \mathbb{R}^{n \times n}$, full matrices

$$
\begin{aligned}
& \widetilde{\mathbf{A}}\left(\rho_{1}, \rho_{2}\right), \widetilde{\mathbf{A}}\left(\overline{\rho_{1}}, \underline{\rho_{2}}\right), \widetilde{\mathbf{A}}\left(\rho_{1}, \overline{\rho_{2}}\right), \widetilde{\mathbf{A}}\left(\overline{\rho_{1}}, \overline{\rho_{2}}\right) \in \mathbb{R}^{n \times n} \\
& \widetilde{\mathbf{B}}\left(\underline{\rho_{1}}, \underline{\rho_{2}}\right), \widetilde{\mathbf{B}}\left(\overline{\rho_{1}}, \underline{\rho_{2}}\right), \widetilde{\mathbf{B}}\left(\underline{\rho_{1}}, \overline{\rho_{2}}\right), \widetilde{\mathbf{B}}\left(\overline{\rho_{1}}, \overline{\rho_{2}}\right) \in \mathbb{R}^{n \times n_{y}} \\
& \widetilde{\mathbf{C}}\left(\underline{\rho_{1}}, \underline{\rho_{2}}\right), \widetilde{\mathbf{C}}\left(\overline{\rho_{1}}, \underline{\rho_{2}}\right), \widetilde{\mathbf{C}}\left(\underline{\rho_{1}}, \overline{\rho_{2}}\right), \widetilde{\mathbf{C}}\left(\overline{\rho_{1}}, \overline{\rho_{2}}\right) \in \mathbb{R}^{n_{u} \times n}
\end{aligned}
$$

and $\gamma \in \mathbb{R}^{+*}$ solving the following problem:

$$
\begin{aligned}
\gamma^{*}=\min & \\
\text { s.t. } & \left.(23)\right|_{\underline{\rho_{1}}}, \underline{\rho_{2}} \\
\text { s.t. } & (23) \mid \overline{\rho_{1}}, \overline{\overline{\rho_{2}}} \\
\text { s.t. } & (23) \mid \overline{\rho_{1}}, \underline{\rho_{2}} \\
\text { s.t. } & (23) \mid \overline{\rho_{1}}, \overline{\rho_{2}}
\end{aligned}
$$

\subsubsection{Proposition: Reconstruction $-\mathcal{H}_{\infty}$ LMI based VDSC design.}

If such $K\left(\rho_{1}, \rho_{2}\right)$ controller exists (Feasibility proposition), the controller reconstruction is obtained solving the following system of equations at each vertices of the polytope, i.e.:

$$
\begin{aligned}
& \text { solve (25) }\left.\right|_{\underline{\rho_{1}}, \underline{\rho_{2}}} \\
& \text { (25) }\left.\right|_{\underline{\rho_{1}}, \overline{\rho_{2}}} \\
& \text { (25) } \mid \overline{\overline{\rho_{1}}}, \underline{\rho_{2}} \\
& \text { (25) }\left.\right|_{\overline{\rho_{1}}, \overline{\rho_{2}}}
\end{aligned}
$$

where $M$ and $N$ are defined such that $M N^{T}=I-X Y$ which may be chosen by applying a singular value decomposition and a Cholesky factorization, as follows:

(1) Singular value decomposition: $I-X Y=U \Sigma V^{T}$ (where $U$ and $V$ are unitary matrices, and $\Sigma$, is a diagonal matrix).

(2) Cholesky factorization: $\Sigma=R^{T} R$, (where $R$ is a real upper triangular matrix). Therefore, $I-X Y=U R R^{T} V^{T}$

(3) Then, one can choose, $M=U R^{T}$ and $N=V R^{T}$

For further details on the LMI, reader is invited to refer to the contributive work of $[29,35-37,41]$. Note that proofs, numerical issues to improve matrix conditioning are also provided in [25]. 


\section{2. $\quad$ Synthesis results}

By solving LMI $(23)$ for $\left\{\rho_{1}, \rho_{2}\right\}=\left[\underline{\rho_{1}}, \overline{\rho_{1}}\right] \times\left[\underline{\rho_{2}}, \overline{\rho_{2}}\right]=[0,1] \times[0,1]$, with YALMIP parser [42] and SeDuMi solver [43], one obtains the following sensitivity functions (Figures 4, 5 and 6) for the LTI and LPV problem (for the LTI controller: $\gamma^{*}=$ 0.5945, and for the LPV controller: $\left.\gamma^{*}=0.6820\right)$.

Remark: The LTI controller is obtained by synthesizing the $\mathcal{H}_{\infty}$ controller on the generalized plant $\Sigma_{g}$ with the same weight filters but with a LTI controller structure (K) such as:

$$
\left\{\begin{aligned}
\dot{x}_{c}(t) & =A_{c} x_{c}(t)+B_{c} e_{\dot{\psi}}(t) \\
{\left[\begin{array}{c}
\delta^{*}(t) \\
T_{b_{r l}}^{*}(t) \\
T_{b_{r r}}^{*}(t)
\end{array}\right] } & =C_{c} x_{c}(t)
\end{aligned}\right.
$$
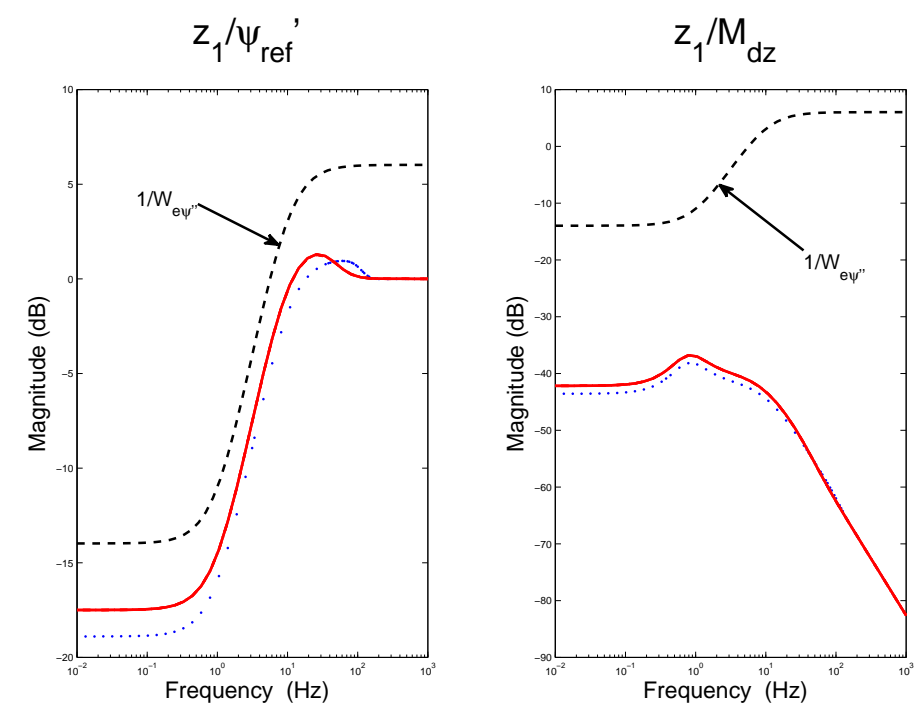

Figure 4. $z_{1}$ controlled output: LTI (blue dotted), LPV with $\rho_{1}=0$ (red solid) and LPV with $\rho_{1}=1$ (red dash-dotted) synthesis results. Weighting functions (black dashed) of $1 / W_{e_{\dot{\psi}}}$.

On these Bode diagrams it is interesting to make the following remarks:

- The yaw rate error signal ( $z_{1}$, Figure 4$)$ for both the LTI and LPV controllers is well attenuated and small yaw rate error is guarantee for low frequencies. Moreover peak frequency is well limited.

- The braking control signal $\left(z_{2}\right.$, Figure 5$)$ rolls off after frequencies higher than $f_{2}$ (actuator bandwidth). Consequently actuator is preserved from high frequencies.

- The output performance signals $z_{1}$ and $z_{2}$ are very similar between the LTI and LPV design (see Figures 4 and 5). This constatation shows that the proposed LPV design does not introduces too much conservatism (remember also that the attenuation level for the LTI controller is: $\gamma^{*}=0.5945$, and for the LPV controller: $\gamma^{*}=0.6820$ ).

- The steering output signal $z_{3}$ of the LPV controller is drastically varying for $\rho_{1}=0$ or $\rho_{1}=1$ (see Figure 6). Recall that when $\rho_{1}=1$ the steering system is used, while $\rho_{1}=0$ means that the steering action is forbidden (therefore, linear results are consistent with the design objectives). As a consequence, when $\rho_{1}=0$, the steering signal is not used. Nevertheless, when $\rho_{1}=1$, the $z_{3}$ signal 

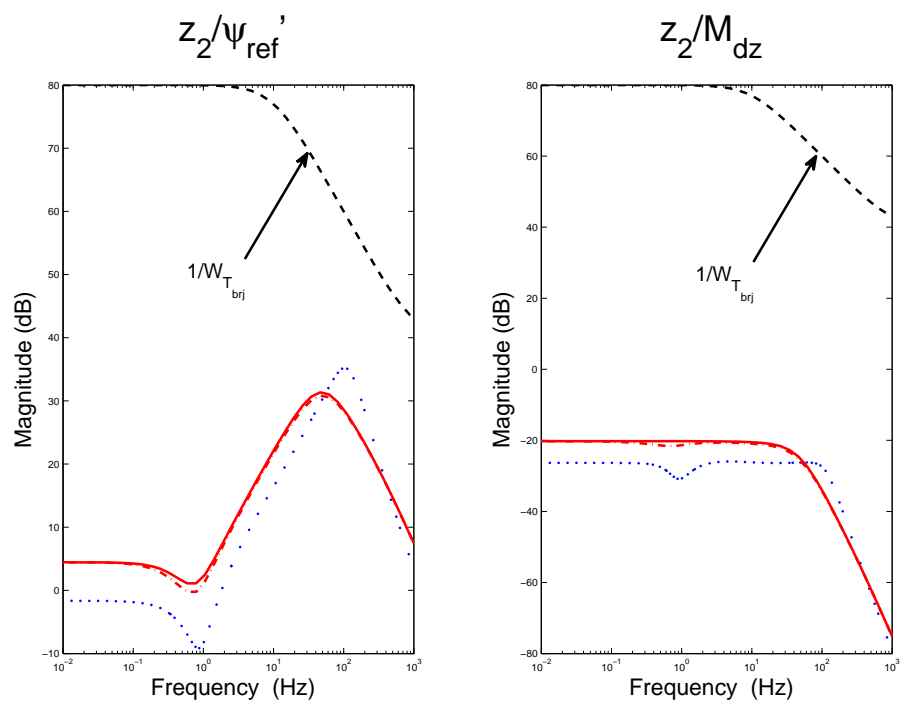

Figure 5. $z_{2}$ controlled output: LTI (blue dotted), LPV with $\rho_{1}=0$ (red solid) and LPV with $\rho_{1}=1$ (red dash-dotted) synthesis results. Weighting functions (black dashed) of $1 / W_{T_{b_{r j}}}$.
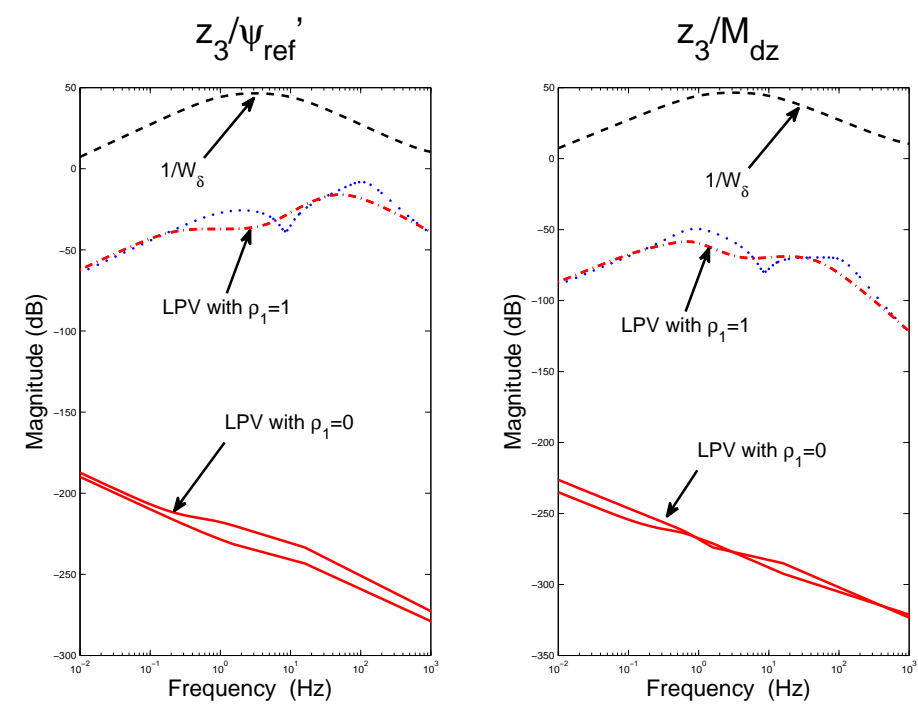

Figure 6. $z_{3}$ controlled output: LTI (blue dotted), LPV with $\rho_{1}=0$ (red solid) and LPV with $\rho_{1}=1$ (red dash-dotted) synthesis results. Weighting functions (black dashed) of $1 / W_{\delta}$.

of the LPV controller is very similar to the LTI one, which is also consistent with the considered performance objective.

- For both LTI and LPV (when $\left.\rho_{1}=1\right)$ control strategies, the steering control $\left(z_{3}\right.$, Figure 6 ) acts on the specified frequency range (between $f_{3}$ and $f_{4}$ ) in order to avoid driver unlikely interaction and preserve actuator bandwidth. 


\subsection{VDSC implementation}

As long as the polytopic design has been used for synthesis, the controller implemented has the following form:

$$
\begin{aligned}
{\left[\delta^{*} T_{b_{r l}}^{*} T_{b_{r r}}^{*}\right]^{T} } & =\rho_{1} \rho_{2} K\left(\overline{\rho_{1}}, \overline{\rho_{2}}\right) \\
& +\left(1-\rho_{1}\right) \rho_{2} K\left(\overline{\rho_{1}}, \rho_{2}\right) \\
& +\rho_{1}\left(1-\rho_{2}\right) K\left(\underline{\rho_{1}}, \overline{\overline{\rho_{2}}}\right) \\
& +\left(1-\rho_{1}\right)\left(1-\overline{\rho_{2}}\right) K\left(\underline{\rho_{1}}, \underline{\rho_{2}}\right)
\end{aligned}
$$

where $K\left(\rho_{1}, \rho_{2}\right)$ are the solutions of the polytopic problem evaluated at each vertices. Note that the gain-scheduled controller results to be a convex combination of four LTI controllers of dimension $n=8$. For implementation purpose, controller order reduction may be applied by using e.g. Hankel singular values (see [44]). Then, discretization of the LPV controller should be applied using specific technique (see very interesting work of [45]).

\subsection{ABS: Local rear ABS controller ([5])}

The previous controller only handles the fact that the braking torque must be positive and actuator dynamical limits preserved, but does not guarantee that the slip ratio is kept low (to avoid wheel locking, leading to loss of manoeuvrability). To make the solution complete, a local ABS strategy is also implemented. Since the synthesis is performed assuming linear tire stiffness, a local controller is essential to prevent from too high braking torque that would lead to slipping situations. In this paper we use the ABS sliding-mode based control law given in $[4,5]$ which exhibits good robustness properties w.r.t. actuator bandwidth, road type and measurement noise and allows to handle the compromise between wheel deceleration low performance and poor slip estimation. This algorithm is not described here but simply briefly recalled.

This strategy, called Mixed Slip and Deceleration (MSD) [4], consists in regulating $\epsilon$, a convex combination of the wheel slip ratio $\lambda$ and of the normalized linear wheel deceleration $\eta=-\frac{\dot{\omega} R}{g}$ measurements around a user defined set point $\epsilon^{*}$ (interested reader should also refer to [5] and author comments [46]).

$$
\epsilon=\alpha \lambda-(1-\alpha) \eta \text { where } \alpha \in[0 ; 1]
$$

According to this MSD measure, the error $\epsilon^{*}-\epsilon$ is regulated using a sliding mode controlled providing a braking torque $\widetilde{T_{b_{r j}}^{A B S}}$ ensuring slip stability. Then, to be integrated to the proposed VDSC structure, one just modifies this control law as:

$$
\widetilde{T_{b_{r j}}^{*}}=\min \left(T_{b_{r j}}^{*} \widetilde{T_{b_{r j}}^{A B S}}\right)
$$

which simply means that the braking torque reference applied on the actuator is the one provided by the VDSC in all situations and ABS one when $T_{b_{r j}}^{*}$ became too high (see also Figure 2). 


\section{Performance analysis and controller validation}

In this section, the proposed gain-scheduled VDSC controller performances are evaluated and compared to other strategies. Note that all validations have been carried on the full vehicle nonlinear model presented in Section 2, which has been validated on a real car (see [25]). For comparison purpose and to validate the interest of the new gain-scheduled VDSC structure (main contribution of the paper), the following time and frequency domain numerical simulations are performed:

(1) First, frequency domain simulations and performance index are computed (see subsections 6.2 and 6.3):

- On the first set of frequency domain experiments, the interest of the combined use of brake and steer actuators with respect to the brake only is illustrated (subsection 6.2).

- In the second set of experiments, the interest of the use of a gain scheduled strategy (LPV) with respect to an LTI one is illustrated (subsection 6.3).

(2) Secondly, time domain simulations of typical driving simulations are performed on different road surfaces to analyze actuator behavior and illustrate the controller improvements through more explicit simulations (subsection $6.4)$.

All simulations are performed on the complete nonlinear vehicle model presented in Section 2. The implementation scheme, given on Figure 2, includes the ABS controller.

\subsection{Performance index definition}

For performance evaluation, a criteria (performance index) is introduced. It is achieved by following a two step procedure (based on a nonlinear frequency response plus a performance index computation):

(1) Compute the Frequency Response of the nonlinear system as follows:

- A sinusoidal signal $u(t)=M_{d z}(t)$ (disturbance moment on the yaw axis of the vehicle) feeds the input of the nonlinear vehicle model, over 5 periods such that:

$$
M_{d z}(t)=A \sin (2 \pi f t)
$$

where $A \in\{1000,2000,5000\} \mathrm{Nm}, f \in[0.1 ; 5] \mathrm{Hz}$.

- The output signals $y(t)$ are measured. Here $y(t)=e_{\dot{\psi}}(t)$ (yaw rate error), $\dot{\theta}(t)$ (roll velocity) and $\beta(t)$ (sideslip angle).

- For each signal the corresponding spectrum $Y(f)$ of $y(t)$ (and $U(f)$ of $M_{d z}(t)$ ) is computed (by mean of a Fourier Transform).

- The power spectral density of $Y(f)$ and $U(f)$ signals are computed; denoted as $G_{y}(f)$ and $G_{u}(f)$.

- For each output signal of interest, the Gain is computed as: $G(f)=$ $G_{y}(f) / G_{u}(f)$. In our case, $G_{e_{\psi}}(f), G_{\dot{\theta}}(f)$ and $G_{\beta}(f)$ signals are obtained. Note that applying this first step procedure on a linear system provides the classical Bode diagram. 
(2) Compute the following performance index:

$$
\begin{aligned}
J_{e_{\psi}} & =\int_{0.1}^{5} \frac{G_{e_{\dot{\psi}}}(f)}{G_{e_{\psi}}^{0}(f)} d f \\
J_{\dot{\theta}} & =\int_{0.1}^{5} \frac{G_{\dot{\theta}}(f)}{G_{\dot{\theta}}^{0}(f)} d f \\
J_{\beta} & =\int_{0.1}^{5} \frac{G_{\beta}(f)}{G_{\beta}^{0}(f)} d f
\end{aligned}
$$

where $J_{e_{\dot{\psi}}}^{0}(f), G_{\dot{\theta}}^{0}(f)$ and $G_{\beta}^{0}(f)$ are the gain of the uncontrolled reference vehicle (Renault Mégane Coup). Consequently, an improvement is achieved when the $J$ performance index is lower than one. Note that the controller is designed in order to enhance yaw rate performances (see Section 3), therefore, the criteria under great interest in this work is the $J_{e_{i}}$ one. The second criteria set, $J_{\dot{\theta}}$ and $J_{\beta}$, is an effect of the vehicle stability control which has to be handled and monitored/minimized. This second criteria is not specifically controlled here but analyzed since it is of great importance in the vehicle stability, especially when the car is subject to high lateral accelerations.

This procedure will be used in the next two subsections to evaluate:

(1) The interest of the join use of the steering and the braking actuators (see $6.2)$.

(2) The benefit the proposed a gain-scheduled LPV controller structure with respect to an LTI control one using both brake and steer (see 6.3).

\subsection{LPV Brake only $\left(\rho_{1}=0\right)$ vs. LPV Brake and Steer $\left(\rho_{1}=1\right)$}

To illustrate the benefit of the combined use of steering and braking actuators to enhance vehicle safety properties, the frequency analysis is done on the full nonlinear vehicle model (see Section 2 ) for varying roads adherence $(\mu)$ and varying disturbance magnitudes $\left(M_{d z}(t)\right)$. The the control improvements are measured by the mean of a the performance index described in 6.1. On Figure 7, $J_{e_{\dot{\psi}}}, J_{\dot{\theta}}$ and $J_{\beta}$ performance index (for varying road surface and disturbance amplitude) of the proposed LPV controller for $\rho_{1}=0$ and $\rho_{1}=1$ are plotted.

By analyzing results plotted on Figure 7, the following comments can be done:

- For all road surface and disturbance magnitude, the controller involving brake and steer actuators $\left(\rho_{1}=1\right)$ provides better results than the controller using the brakes only $\left(\rho_{1}=0\right)$. Additionally, note that the controller using both steering and braking actuators will preserve braking actuators and reduce energy consumption.

- For high $\mu$ situations (e.g. Dry and Cobblestone situations), the increase of the $M_{d z}$ disturbance amplitude does not significantly modify the gain ratio between controller with controller with $\rho_{1}=0$ and controller with $\rho_{1}=1$. As a matter of fact, $J_{e_{\dot{\psi}}}\left(\rho_{1}=1\right) / J_{e_{\psi}}\left(\rho_{1}=0\right), J_{\dot{\theta}}\left(\rho_{1}=1\right) / J_{\dot{\theta}}\left(\rho_{1}=0\right)$ and $J_{\beta}\left(\rho_{1}=1\right) / J_{\beta}\left(\rho_{1}=0\right)$ are almost constant. Note also that the $J$ criteria decreases as the disturbance gain increases; this phenomena is more due to the fact that the uncontrolled vehicle performances are decreased than to a controller improvement.

- More significant, for low $\mu$ situation (e.g. Wet road), it is very interesting to note that the ratios $J_{e_{\psi}}\left(\rho_{1}=1\right) / J_{e_{\psi}}\left(\rho_{1}=0\right), J_{\dot{\theta}}\left(\rho_{1}=1\right) / J_{\dot{\theta}}\left(\rho_{1}=0\right)$ and $J_{\beta}\left(\rho_{1}=\right.$ $1) / J_{\beta}\left(\rho_{1}=0\right)$ is very different than for high $\mu$ situations. More specifically, 


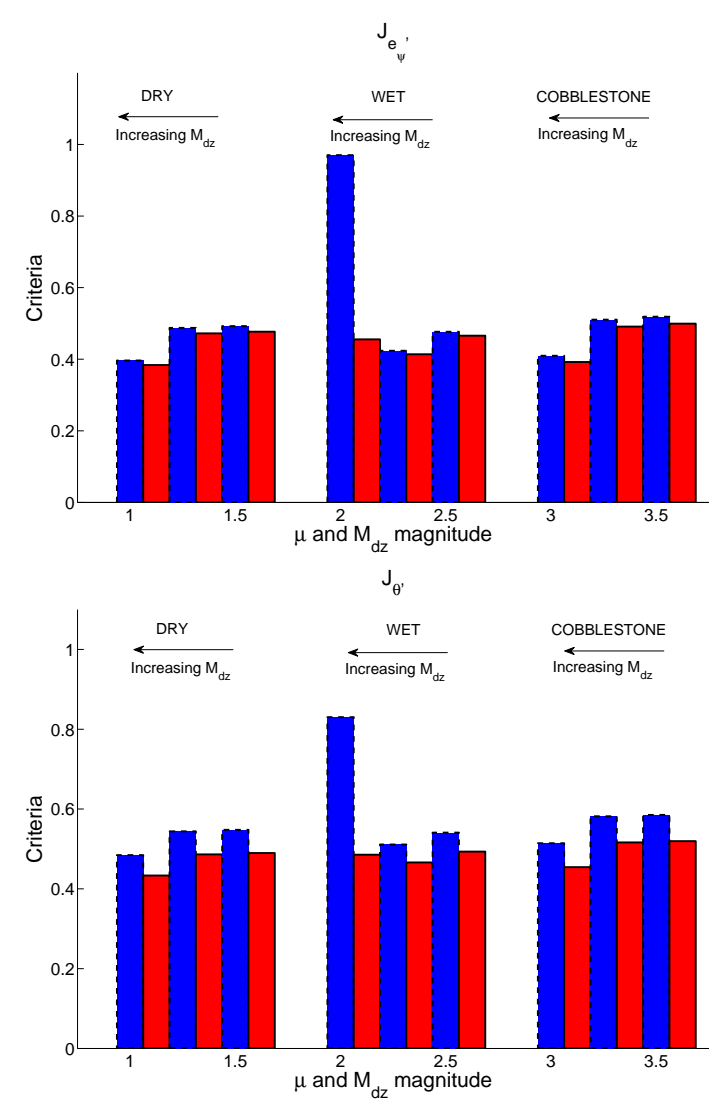

$\beta$

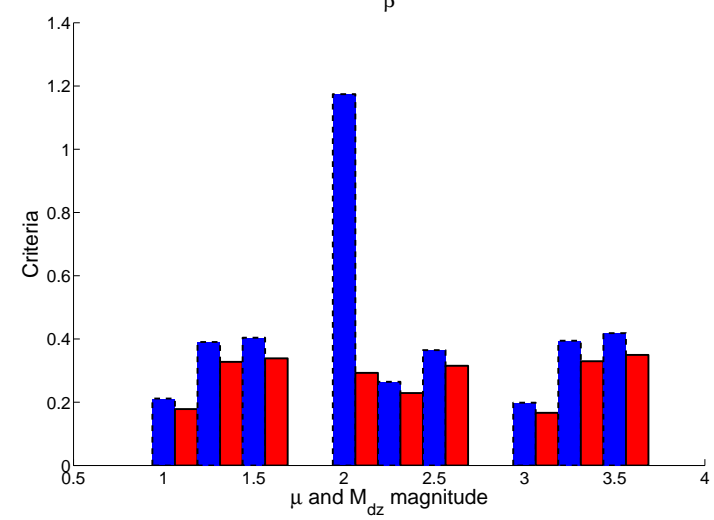

Figure 7. Performance index $J_{e_{\dot{\psi}}}$ (up), $J_{\dot{\theta}}$ (middle) and $J_{\beta}$ (bottom) for varying road adherence $\mu$ (dry, wet, cobblestone) and disturbance amplitude $(A \in\{1000,2000,5000\} \mathrm{Nm})$ with an initial speed of the vehicle: $v_{0}=90 \mathrm{~km} / \mathrm{h}$. Comparison of the LPV control design with $\rho_{1}=0$, i.e. without use of the steering signal (blue and dashed contour) and $\rho_{1}=1$, i.e. with the use of the steering signal (red and solid contour).

improvements obtained by the only brake controller are very bad compared to the one obtained with the join use of brake and steer actuators. This major difference can be explained by the fact that the braking forces saturate and are no longer able to stabilize the vehicle. Therefore, in this situation the use of the steer actuator appears to be essential to keep brake force lower and guarantee vehicle stability while maintaining low sideslip angle and low roll velocity. Note that here, this saturation phenomena is illustrated on an wet road, but also exists on dry and cobblestone road surface (for higher amplitude disturbances).

According to this first set of simulations, it appears that the join use of the steering and braking actuators enhances the vehicle dynamical properties. Moreover, it 


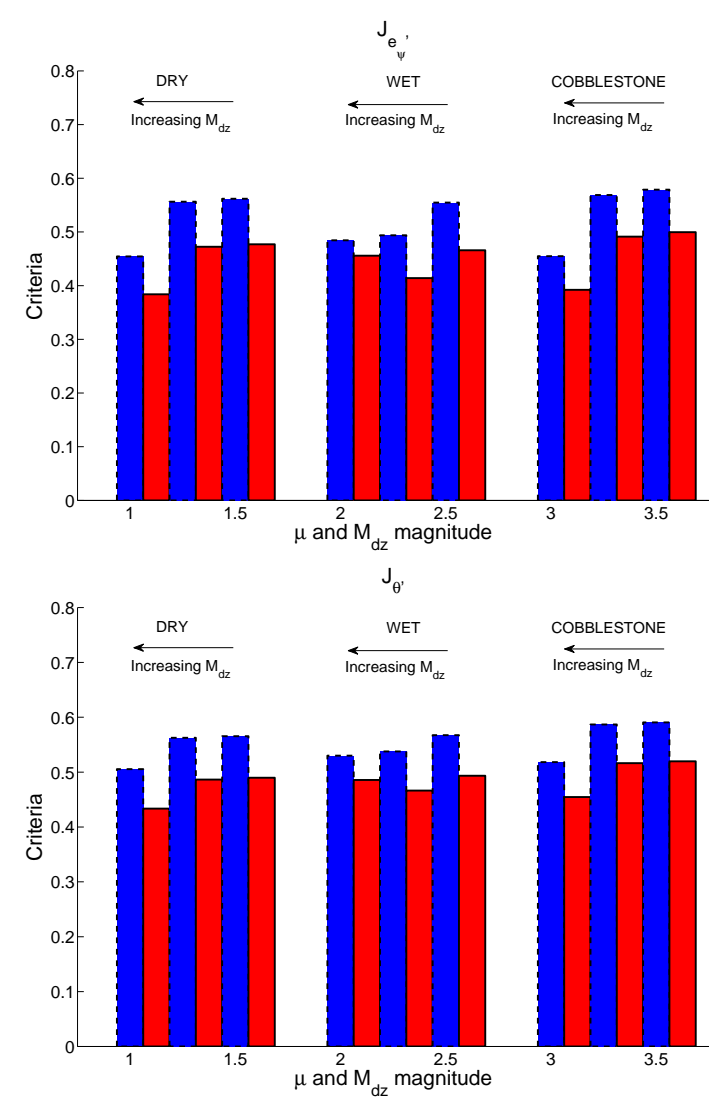

Figure 8. Performance index $J_{e_{\dot{\psi}}}$ (up) and $J_{\dot{\theta}}$ (bottom) for varying road adherence $\mu$ (dry, wet, cobblestone) and disturbance amplitude $(A \in\{1000,2000,5000\} \mathrm{Nm})$ with an initial speed of the vehicle: $v_{0}=90 \mathrm{~km} / \mathrm{h}$. Comparison of the LTI control design (blue and dashed contour) and the LPV control design with $\rho_{1}=1$, i.e. with the use of the steering signal (red and solid contour).

also appears that the steer actuator is essential when high disturbances occurs on low $\mu$ road surface (note that this phenomena also occurs for increasing vehicle velocity). This observation is consistent with previous literature results [20, 22, 47]. From now one, comparisons will only involve controllers using both brake and steer actuators.

\subsection{LTI vs. LPV (with join Brake and Steer control)}

In the previous subsection, the improvement of a join control of brake and steer to enhance vehicle stability is demonstrated through nonlinear frequency experiments performed on different road type and for varying disturbance amplitude. Here we aim at demonstrate that the proposed gain-scheduled VDSC, which handles the braking limitations (e.g. positive torque only), involving braking and steering (e.g. $\rho_{1}=1$ ), provides better results than an LTI controller (synthesized with the same weighting functions but a fixed state space representation, using the same actuators). On Figure $8, J_{e_{\dot{\psi}}}$ and $J_{\dot{\theta}}$ performance index are plotted ( $\beta$ analysis is let for space reasons, but will be analyzed specifically in time simulations, in Section 6.4).

By analyzing results of Figure 8, the following comments can be done:

- Both controllers (LPV and LTI) enhance vehicle dynamics properties (i.e. Performance index lower than one).

- For all road adherence and disturbance magnitude, the LPV gain-scheduled con- 
troller involving brake and steer $\left(\rho_{1}=1\right)$ provides better results than the LTI controller.

- The ratios between LTI and LPV improvements are slightly better as the $\mu$ value is low. This phenomena may be due to the fact that the brake forces saturate faster than on high road adherence. Therefore, considering the LTI controller, the braking torque would saturate when negative torque are required (since this demand is never possible) and when high brake demand is required (due to low road friction) as well. Therefore, in this low friction situation, the LPV controller shows to become a real necessity.

To more accurately underline theses results, Figures 9 shows the frequency responses of the controlled system on a wet road (very low road friction force) for a vehicle running at $50 \mathrm{~km} / \mathrm{h}$ and $120 \mathrm{~km} / \mathrm{h}$ (for $e_{\dot{\psi}}, \dot{\theta}$ and $\beta$ signals).

On these frequency plots, it is clear that the LPV control design provides better yaw rate tracking behavior than the LTI one in all situations. Concerning the roll velocity and sideslip angle, a global improvement is achieved but not locally around certain frequencies. By the way, since this variable is not specifically controlled (i.e. included in the control design) it simply is an effect of the yaw rate control. in conclusion to this frequency results, the presented contribution using gain-scheduled VDSC shows to be more efficient than LTI VDSC (on varying frequencies, road surface and vehicle velocities). Note that if an accurate sideslip angle observer is available, the use of $\beta$ in the synthesis step may be done to enhance the results (but this is not the focus of the present paper)

\subsection{Nonlinear time domain performance analysis}

Since now, all validations where performed using frequency domain simulations which is crucial for performance evaluation but may not illustrate clearly the behavior on real dangerous driving simulations. In this section, simulations on a critical driving situation (quick line change, obstacle avoidance) are performed to illustrate the benefit of the control design (note that in this simulation, no driver model is considered, the steering input signal is provided as on Figure 10). More than performance demonstration, the aim here is principally to show that the controller output signals fulfills the brake constrain, which is one of the contributions of this paper. The following two situations are computed:

- Simulation 1 - LPV $\rho_{1}=1$ vs. LTI (both using brake and steer actuators): Vehicle runs at $90 \mathrm{~km} / \mathrm{h}$ on a WET road surface and driver performs a line change manoeuver.

- Simulation 2 - LPV $\rho_{1}=1$ vs. LTI (both using brake and steer actuators): Vehicle runs at $70 \mathrm{~km} / \mathrm{h}$ on an ICY road surface and driver performs a line change manoeuver.

On both simulations, the driver steering input signal is given as on Figure 10. This manoeuver is equivalent to an obstacle avoidance. Vehicle trajectories for both simulations are given on Figure 11.

\subsubsection{Dynamic performance analysis (Simulation 1 vs. Simulation 2)}

According to this simulation setup, the following performance curve results are obtained, which presents the vehicle behavior: yaw rate error behavior on Figure 12 and sideslip response on Figure 13 (see vehicle also trajectory on Figure 11).

According to Figure 11 and 12, the following comments can be done:

- On both Simulation 1 and 2, the gain-scheduled VDSC controller (with $\rho_{1}=1$ ) shows to highly enhance the yaw rate tracking error, even more than the LTI 

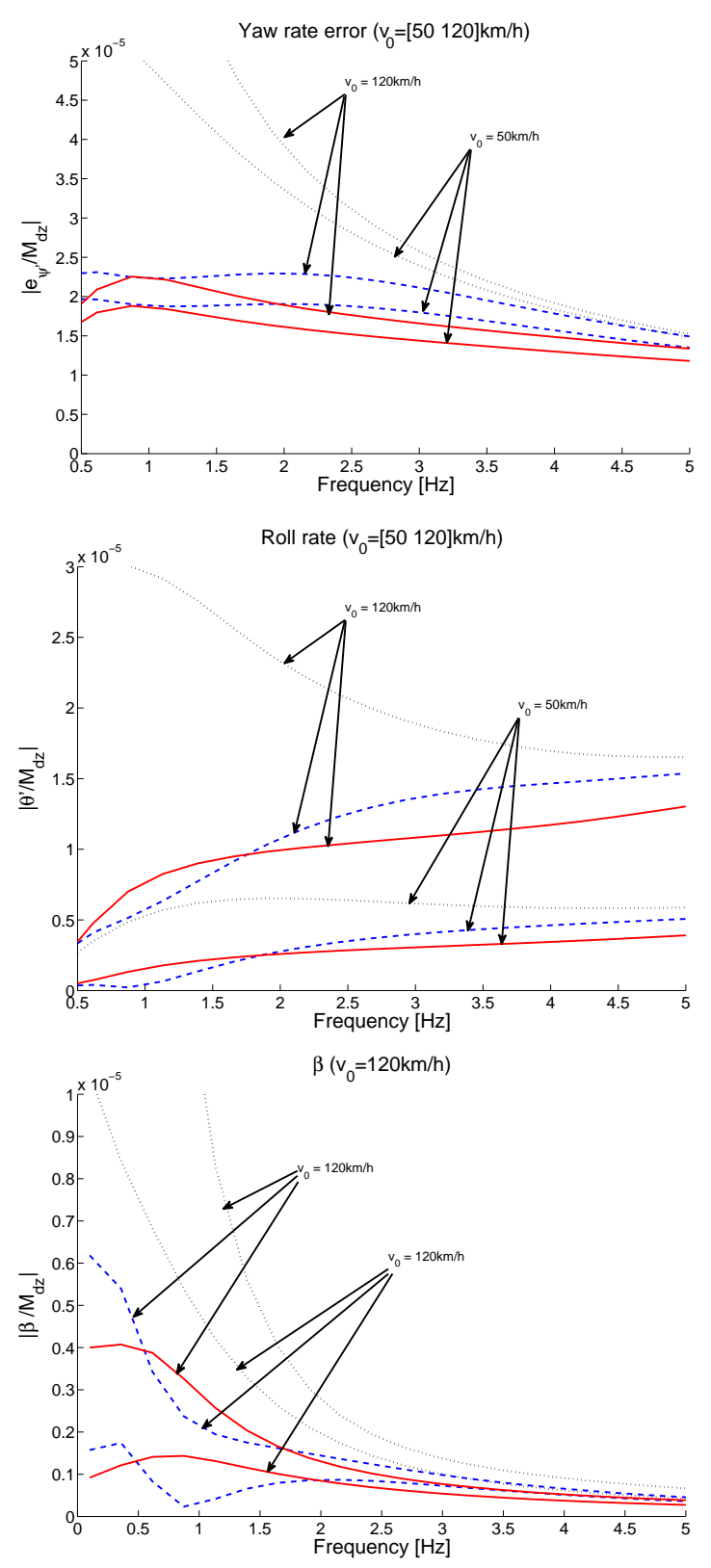

Figure 9. Frequency responses of $e_{\dot{\psi}} / M_{d z}$ (up) and $\dot{\theta} / M_{d z}$ (bottom) for WET road with initial speed of the vehicle: $v_{0}=[50,120] \mathrm{km} / \mathrm{h}$ and disturbance amplitude of $2000 \mathrm{Nm}$. Comparison of the Uncontrolled (black dotted) LTI control design (blue dashed) and the LPV control design with $\rho_{1}=1$, i.e. with the use of the steering signal (red solid).

controller (left frame of Figures 11 and 12).

- Moreover, on Simulation 2 (right frame of Figures 11, 12 and 13, where low $\mu$ is considered), the gain-scheduled controller shows to keep vehicle trajectory more acceptable than the LTI design. Indeed the $\beta$ angle is kept acceptable (lower than $7 \mathrm{deg}$ ) and yaw rate tracking enhanced (i.e. $e_{\dot{\psi}}$ reduced).

\subsubsection{Control signal analysis (Simulation 1 only)}

Since the main contribution of this works concerns the controller structure which handles brakes limitations (i.e. the brake saturation), control signals of Simulation 1 are more deeply analyzed in this part. Figure 14 shows the additive steering signal (generated by the controller and provided by the actuator). 


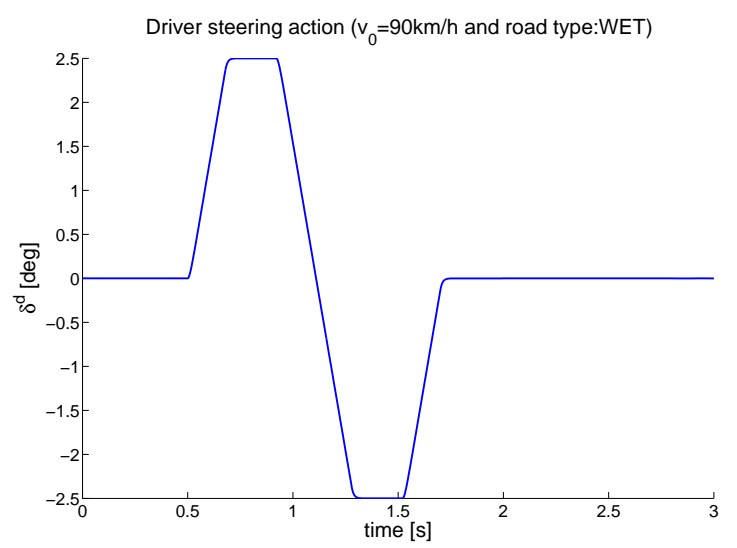

Figure 10. Driver steering input signal (avoidance manoeuver).
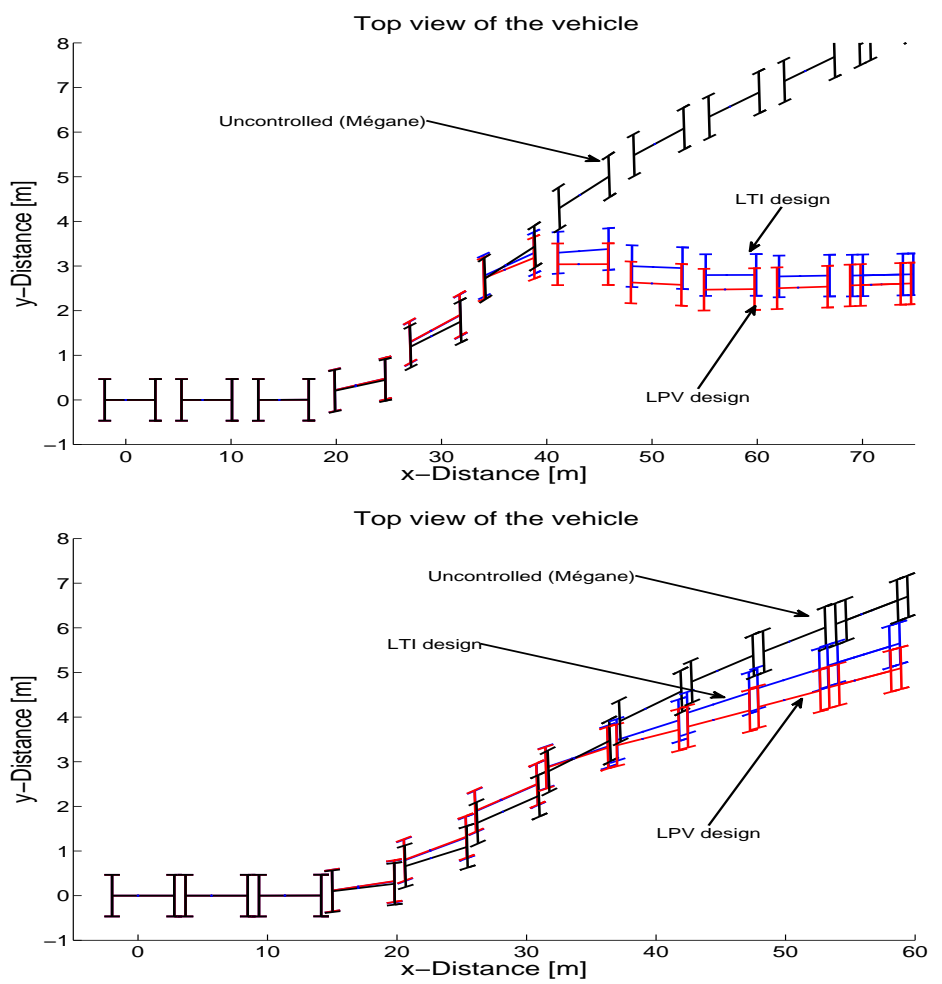

Figure 11. Vehicle trajectory: Simulation 1 (left). Simulation 2 (right). Uncontrolled 'Mégane' (black), 'LTI' (blue), and 'VDSC with steer' (red).
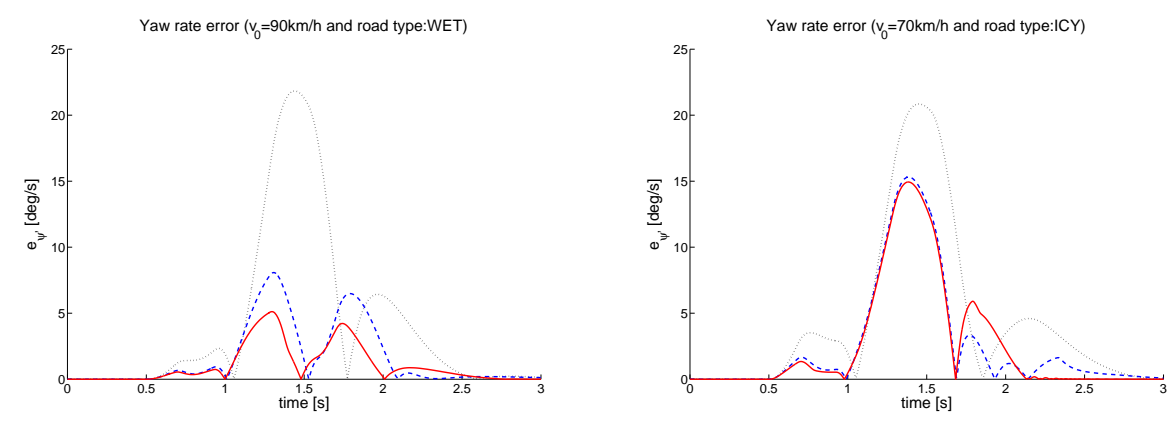

Figure 12. Yaw rate error $\left(e_{\dot{\psi}}\right)$ : Simulation 1 (left). Simulation 2 (right). For the uncontrolled 'Mégane' (blue pointed thin), 'LTI VDSC' (blue dashed thick), 'LPV VDSC with $\rho_{1}=1$ ' (red solid thick). 

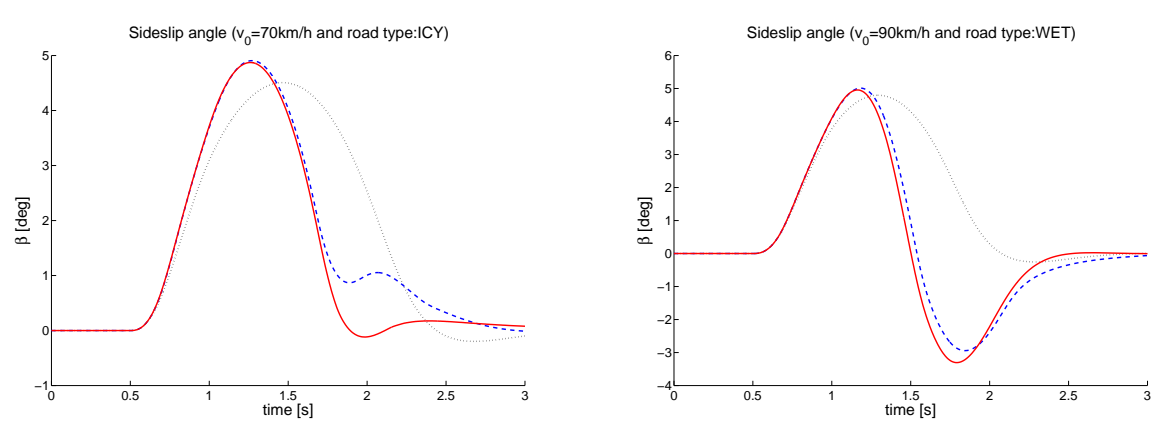

Figure 13. Sideslip $(\beta)$ : Simulation 1 (left). Simulation 2 (right). For the uncontrolled 'Mégane' (blue pointed thin), 'LTI VDSC' (blue dashed thick), 'LPV VDSC with $\rho_{1}=1$ ' (red solid thick).

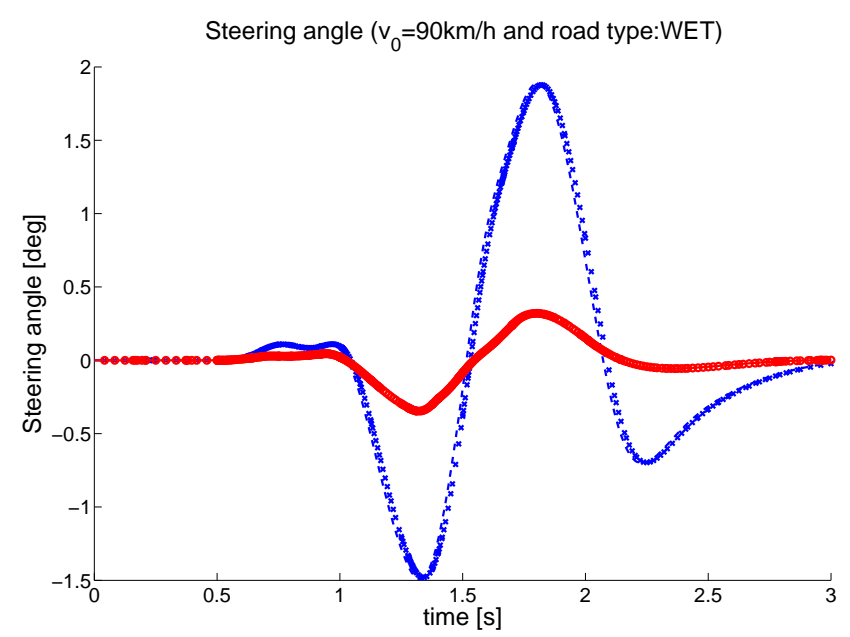

Figure 14. Steering control signals of Simulation 1. Steering signal provided by the 'LTI VDSC': controller output (blue dashed) and by the actuator output (blue cross). Steering signal provided by the 'LPV VDSC with $\rho_{1}=1$ ': controller output (red solid) and actuator output (red round).

From Figure 14, it is obvious that the LTI design requires more additive steering control. This behavior is due to fact that brake its signal is reduced by two since acceleration of one wheel if forbidden. Conversely, the proposed VDSC using the LPV approach does not requires high additive steering to keep vehicle dynamics, therefore. Therefore, it preserves for steering actuator saturation. Figure 15 shows the braking signals (generated by the controller and provided by the saturated actuator).

According to Figure 15, it is interesting to remark that, the gain-scheduled VDSC control left and right torques are always positive (i.e. only provide braking action) while the LTI ones are both positive and negative (which are impossible to achieve by the considered actuators). Therefore, LPV braking actuators are not always saturating and it results in an improved efficiency and avoid a classical trial and error approach to validate control design. From these plots it result that the LPV design avoids actuator saturation while the LTI one leads to impossible control signals (see Figure 15). On Figure 16, the $\rho_{2}$ parameter is also plotted to show the scheduling parameter variations. On Figure 15-bottom, it is also notable that between 1 and 1.5s (at its peak value), the brake torque provided by the LPV controller is saturated by the ABS algorithm, e.g. the ABS is activated to handle slip stability. This remarks shows the nice integration of the proposed design with the ABS algorithm.

Finally, from a general point of view, the following comments may be done: 

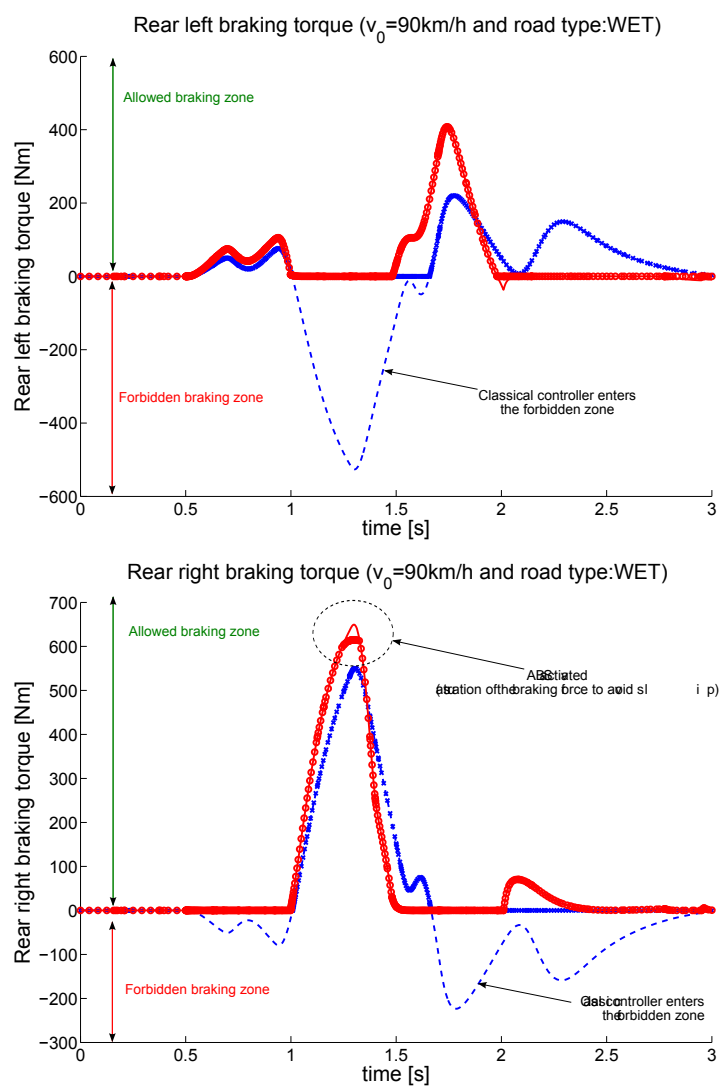

Figure 15. Braking control signals of Simulation 1 (rear left brake, top / and rear right brake, bottom). Brake torque signal provided by the 'LTI VDSC': controller output (blue dashed) and by the actuator output (blue cross). Brake torque signal provided by the 'LPV VDSC with $\rho_{1}=1$ ': controller output (red solid) and actuator output (red round).

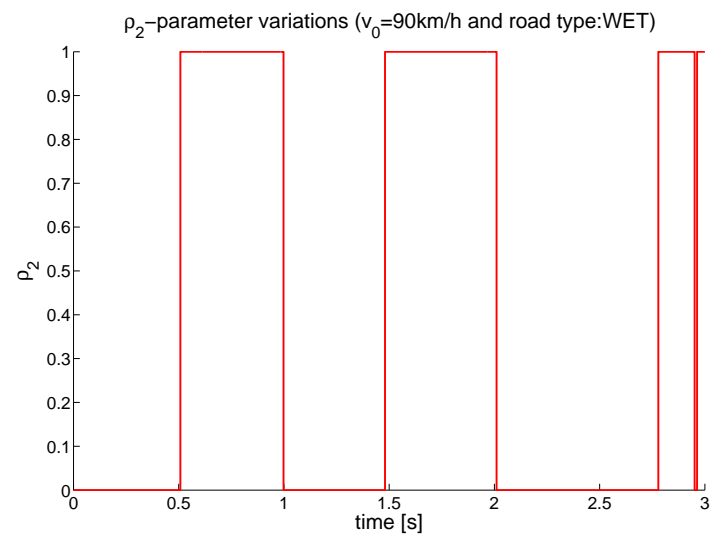

Figure 16. $\rho_{2}$ parameter variation (used to ensure braking torque constraints).

- Both LTI and LPV VDSC enhance the yaw rate tracking. But still the LPV one provides better results (see Figure 12).

- The LTI controller generates both positive and negative braking toques, then the saturation clearly deteriorates the performances and the controller is not adapted to the system constraints. While the LPV controller only provides positive braking torques, which are achievable by the considered actuators. Therefore, the new proposed controller fits to the actuator constraints. Still some peaks appears when scheduling, but theses discontinuities do not affect the closed-loop 
stability due to the LPV design (see Figure 15).

Authors stress that usually, in the literature, the brake control is handled trough a differential mechanism, while the here proposed approach is original in the sense where brakes are treated independently and saturation effect is treated through an original LPV design, which is a contribution with respect to previous works, allowing different left / right brake control in complex situations.

\section{Conclusions and Discussions}

In this paper we address the problem of yaw vehicle dynamical stability control (while minimizing sideslip and roll velocity), which is one of the most critical point for light vehicles. The proposed MIMO gain-scheduled VDSC solution involves an active steering and rear braking actuators. The main contribution and innovation is to propose a methodology to design a controller tacking into consideration actuators limitations and varying performance requirements, i.e.:

- The steering control only acts in a given frequency range, corresponding to an action non achievable by a normal driver (frequencies $>1 \mathrm{~Hz}$ ), and limited by the steering actuator bandwidth. Additionally, the steering actuator can be activated by a simple parameter adjustment $\left(\rho_{1}\right)$ in order to use this actuator as a last solution (if a critical situation is detected). Note that the critical situation detection is not covered in this paper; but in [23] a solution to tune $\rho_{1}$ using the braking efficiency measure is also proposed.

- Thanks to the $\rho_{2}$ parameter and a controller particular structure, the braking torque control signal is guaranteed to be always positive, i.e. only braking action is possible (no acceleration), which is a contribution, especially in the linear robust framework where saturations are often treated using anti-windup design (see e.g. recent results $[48,49]$ ). This approach avoids in an original and efficient way the usual differential braking solution used in the literature.

Additionally, in this paper the proposed design is integrated with a recently developed local ABS control, making the solution fully implementable on a real vehicle (handling slip and yaw dynamics problems without requiring any on-line optimization procedure). Moreover, since the control design is performed in the LPV robust framework, internal stability and performances are guaranteed for the entire parameter varying set.

The main contribution in this control design relies in the fact that control amplifications due to saturations are avoided and system properties are exploited to design a controller in a smarter way. Performances result to be more robust and efficient in complex driving situations. Therefore, according to the authors, the main contributions of this paper is the use of a particular controller structure, together with system properties, to design a robust controller that handles steering/braking actuators constraints in an original way.

The proposed design has been validated on a complex nonlinear vehicle model, with values identified and validated on a real vehicle (see [25]), over different driving situations, using:

- Time and frequency domain simulations

- A frequency performance evaluation index

Results show the efficiency of the control algorithm compared to simpler approaches (e.g. with only braking or with an LTI structure) and presents interesting robustness properties to low road adherence and vehicle velocity variations as well as a 
performance specification and measurement high flexibility.

\section{References}

[1] P. Gáspár, Z. Szabó, J. Bokor, C. Poussot-Vassal, O. Sename, and L. Dugard, Toward global chassis control by integrating the brake and suspension systems, in Proceedings of the 5th IFAC Symposium on Advances in Automotive Control (AAC), August, , Aptos, California, USA, 2007.

[2] M. Denny, The dynamics of antilock brake systems, European Journal of Physics 26 (2005), pp. 1007-1016.

[3] T. Johansen, I. Petersen, J. Kalkkuhl, and J. Ludemann, Gain-scheduled wheel slip control in automotive brake systems, IEEE Transaction on Control System Technology 11 (2003), pp. 799-811.

[4] S. Savaresi, M. Tanelli, and C. Cantoni, Mixed Slip-Deceleration Control in Automotive Braking Systems, ASME Transactions: Journal of Dynamic Systems, Measurement and Control 129 (2007), pp. 20-31.

[5] M. Tanelli, R. Sartori, and S. Savaresi, Combining Slip and Deceleration Control for Brake-by-Wire Control Systems: a Sliding-Mode Approach, European Journal of Control 13 (2007), pp. 593-611.

[6] E. Rossetter and J. Gerdes, A study of lateral vehicle control under a Virtual force framework, in Proceedings of the International Symposium on Advanced Vehicle Control, Hiroshima, Japan, 2002.

[7] J. Ackermann and T. Bunte, Yaw disturbance attenuation by robust decoupling of car steering, in Proceedings of the 13th IFAC World Congress (WC), june, , San Francisco, California, 1996, pp. 1-6.

[8] P. Falcone, F. Borrelli, J. Asgari, H. Tseng, and D. Hrovat, Predictive Active Steering Control for Autonomous Vehicle Systems, IEEE Transaction on Control System Technology 15 (2007), pp. 566580 .

[9] J. Villagra, B. Noveld'Andra, H. Mounier, and M. Pengov, Flatness-Based Vehicle Steering Control Strategy With SDRE Feedback Gains Tuned Via a Sensitivity Approach, IEEE Transaction on Control System Technology 15 (2007), pp. 554-565.

[10] B.A. Guven, T. Bunte, D. Odenthal, and L. Guven, Robust Two Degree-of-Freedom Vehicle Steering Controller Design, IEEE Transaction on Control System Technology 12 (2004), pp. 627-636.

[11] S. Mammar and D. Koenig, Vehicle handling improvement by active steering, Vehicle System Dynamics 38 (2002), pp. 211-242.

[12] M. Canale, L. Fagiano, M. Milanese, and P. Borodani, Robust vehicle yaw control using an active differential and IMC techniques, Control Engineering Practice 15 (2007), pp. 923-941.

[13] P. Gáspár, Z. Szabó, and J. Bokor, Side force coefficient estimation for the design of an active brake, in Proceedings of the IEEE American Control Conference (ACC), june, , Mineapolis, Minesota, 2006, pp. 2927-2932.

[14] T. Acarman, Nonlinear optimal integrated vehicle control using individual braking torque and steering angle with on-line control allocation by using state-dependent Riccati equation technique, Vehicle System Dynamics (2008), p. DOI: 10.1080/00423110801932670.

[15] P. Gáspár, I. Szaszi, and J. Bokor, Rollover stability control for heavy vehicles by using LPV model, in Proceedings of the 1st IFAC Symposium on Advances in Automotive Control (AAC), april, , Salerno, Italy, 2004.

[16] J. Lu and M. DePoyster, Multiobjective Optimal Suspension Control to Achieve Integrated Ride and Handling Performance, IEEE Transaction on Control System Technology 10 (2002), pp. 807-821.

[17] J. Meijaard, A. Savkoor, and G. Lodewijks, Potential for vehicle ride improvement using both suspension and aerodynamic actuator, in Proceedings of the IEEE Internation Symposium on Industrial Electronics (ISIE), june, Dubrovnik, Croatia, 2005, pp. 385-390.

[18] H. Chou and B. Noveld'Andra, Global vehicle control using differential braking torques and active suspension forces, Vehicle System Dynamics 43 (2005), pp. 261-284.

[19] P. Gáspár, Z. Szabó, and J. Bokor, The design of an integrated control system in heavy vehicles based on an LPV method, in Proceedings of the 44th IEEE Conference on Decision and Control (CDC), December, , Seville, Spain, 2005, pp. 6722-6727.

[20] S. Baslamisli, I. Kse, and G. Anlas, Gain Scheduled Integrated Active Steering and Active Differential Control for Vehicle Handling Improvement, Vehicle System Dynamics 41 (2009), pp. 99-119.

21] P. Falcone, F. Borrelli, H. Tseng, J. Asgari, and D. Hrovat, Integrated braking and steering model predictive control approach in autonomous vehicles, in Proceedings of the 5th IFAC Symposium on Advances on Automotive Control (AAC), August, , Aptos, California, 2007.

[22] J. Andreasson and T. Bunte, Global chassis control based on inverse vehicle dynamics models, Vehicle System Dynamics 44 (2006), pp. 321-328.

[23] C. Poussot-Vassal, O. Sename, and L. Dugard, A Global Chassis Controller for Handling Improvements Involving Braking and Steering Systems, in Proceedings of the 47 th IEEE Conference on Decision and Control (CDC), December, , Cancun, Mexico, 2008.

[24] — Robust Vehicle Dynamic Stability Controller Involving Steering and Braking Systems, in Accepted to the 9th European Control Conference (ECC), August, , Budapest, Hungary, 2009.

[25] C. Poussot-Vassal, Robust Multivariable Linear Parameter Varying Automotive Global Chassis Control, PhD Thesis (in English), Grenoble INP, GIPSA-lab, Control System dpt., Grenoble, France, 2008.

[26] A. Zin, O. Sename, P. Gaspar, L. Dugard, and J.Bokor, Robust LPV - $\mathcal{H}_{\infty}$ Control for Active Suspensions with Performance Adaptation in view of Global Chassis Control, Vehicle System Dynamics 46 (2008), pp. 889-912.

[27] C. Poussot-Vassal, O. Sename, L. Dugard, P. Gáspár, Z. Szabó, and J. Bokor, New Semi-active Suspension Control Strategy Through LPV Technique, Control Engineering Practice 16 (2008), pp. 1519-1534.

[28] U. Kiencke and L. Nielsen Automotive Control Systems, , 2000. 
[29] J. Doyle, K. Glover, P. Khargonekar, and B. Francis, State space solution to standard $\mathcal{H}_{2}$ and $\mathcal{H}_{\infty}$ control problems, IEEE Transaction on Automatic Control 34 (1989), pp. 831-847.

[30] B. Guven, L. Guven, and S. Karaman, Robust Yaw Stability and hardware-in-the-loop testing for a road vehicle, IEEE Transaction on Control System Technology 58 (2009), pp. 555-571.

[31] P. Pisu, A. Soliman, and G. Rizzoni, Vehicle chassis monitoring system, Control Engineering Practice 11 (2003), pp. 345-354.

[32] D. Fisher, M. Borner, J. Schmitt, and R. Isermann, Fault detection for lateral vertical vehicle dynamics, Control Engineering Practice 15 (2007), pp. 315-324.

[33] C. Lauwerys, J. Swevers, and P. Sas, Vehicle chassis monitoring system, Control Engineering Practice 13 (2005), pp. 577-586.

[34] P. Apkarian and P. Gahinet, A convex characterization of gain scheduled $\mathcal{H}_{\infty}$ controllers, IEEE Transaction on Automatic Control 40 (1995), pp. 853-864.

[35] C. Scherer, P. Gahinet, and M. Chilali, Multiobjective Output-Feedback Control via LMI Optimization, IEEE Transaction on Automatic Control 42 (1997), pp. 896-911.

[36] P. Gahinet, P. Apkarian, and M. Chilali, Affine parameter-dependent Lyapunov functions and real parametric uncertainty, IEEE Transaction on Automatic Control 41 (1996), pp. 436-442.

[37] P. Apkarian, P. Gahinet, and G. Beker, Self-scheduled $\mathcal{H}_{\infty}$ control of linear parameter-varying systems: A design example, Automatica 31 (1995), pp. 1251-1262.

[38] P. Apkarian, P. Gahinet, and J. Biannic, Self-scheduled $\mathcal{H}_{\infty}$ control of a missile via LMIs, in Proceedings of the 33rd IEEE Conference on Decision and Control (CDC), December, , Lake Buena Vista, Florida, 1994, pp. 3312-3317.

[39] C. Souzade and A. Trofino, Gain scheduled $\mathcal{H}_{2}$ controller synthesis for linear parameter varying systems via parameter-dependent Lyapunov functions, International Journal of Robust and Nonlinear Control 16 (2005), pp. 243-257.

[40] F. Bruzelius, S. Pettersson, and C. Breitholz, Linear parameter varying descriptions of nonlinear systems, in Proceedings of the IEEE American Control Conference (ACC), july, , Boston, Massachusetts, 2004, pp. 1374-1379.

[41] J. Shamma and M. Athans, Guaranteed Properties of Linear Parameter Varying Gain Scheduled Control Systems, Automatica 27 (1991), pp. 559-564.

[42] J. Lofberg, YALMIP : A Toolbox for Modeling and Optimization in MATLAB, in Proceedings of the CACSD Conference, Taipei, Taiwan, 2004.

[43] J.F. Sturm, Using SeDuMi 1.02, a MATLAB toolbox for optimization over symmetric cones, Optim. Methods Softw. 11/12 (1999), pp. 625-653 Interior point methods.

[44] K. Glover, All Optimal Hankel Norm Approximation of Linear Multivariable Systems, and Their $\mathcal{L}_{\infty}$ error Bounds, International Journal Control 39 (1984), pp. 1145-1193.

[45] R. Toth, F. Felici, P. Heuberger, and P.V. Hofder, Crutial aspects of zero-order hold LPV state-space system discretization, in Proceedings of the 17th IFAC World Congress (WC), July, , Seoul, South Korea, 2008.

[46] C. Poussot-Vassal, Discussion paper on: "Combining Slip and Deceleration Control for Brake-by-Wire Control Systems: a Sliding-Mode Approach", European Journal of Control 13 (2007), pp. 612-615.

[47] P. Falcone, M. Tufo, F. Borrelli, J. Asgari, and H. Tseng, A Linear Time Varying Model Predictive Control Approach to the Integrated Vehicle Dynamics Control Problem in Autonomous Systems, in Proceedings of the 46th IEEE Conference on Decision and Control (CDC), December, , New Orleans, Louisiane, USA, 2007.

[48] C. Roos and J.M. Biannic, A convex characterization of dynamically-constrained anti-windup controllers, Automatica 44 (2008), pp. 2449-2452.

[49] J.M. Biannic and S. Tarbouriech, Optimization and implementation of dynamic anti-windup compensators with multiple saturations in flight control systems, Control Engineering Practice (2009), p. doi:10.1016/j.conengprac.2008.11.002. 